\title{
Pre-Objective Polygonal Scanning
}

\section{Gerald F. Marshall}

Consultant in Optics

Niles, Michigan, USA

\section{CONTENTS}

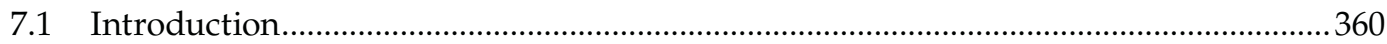

7.1.1 Equations and Coordinates of a Polygonal Scanning System ............................... 361

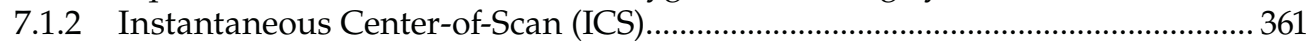

7.1.3 Stationary Ghost Images Outside the Image Format............................................ 361

7.2 Equations and Coordinates of a Polygonal Scanning System ….................................. 361

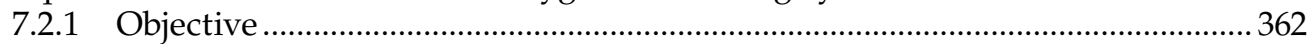

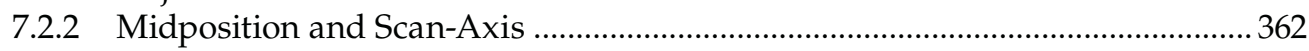

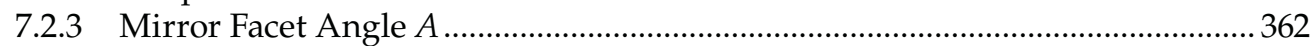

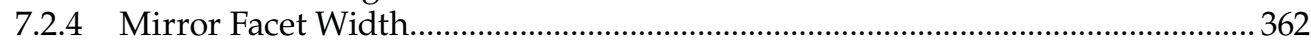

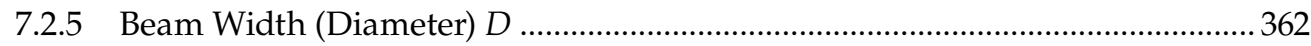

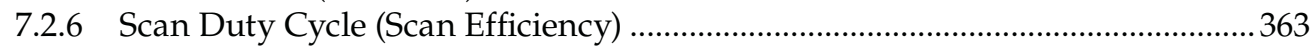

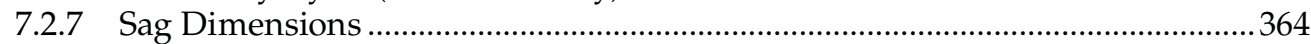

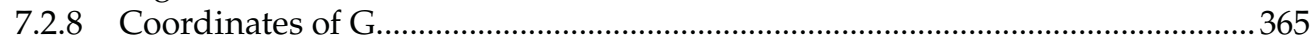

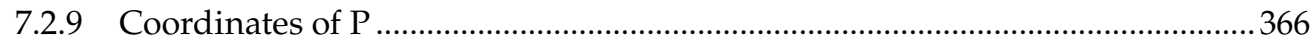

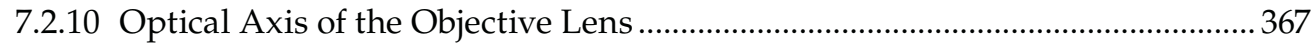

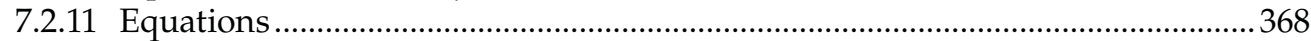

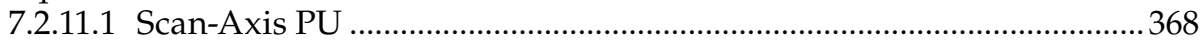

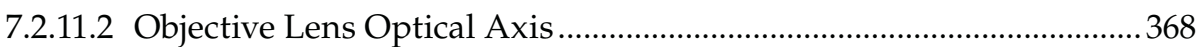

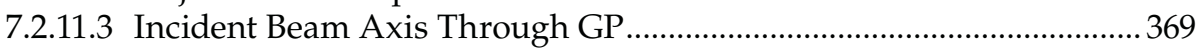

7.2.11.4 Mirror Facet Bisector and Normal.......................................................... 369

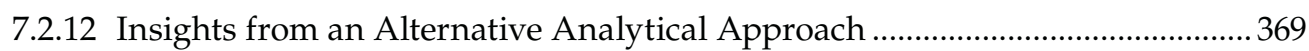

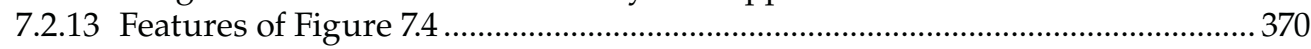

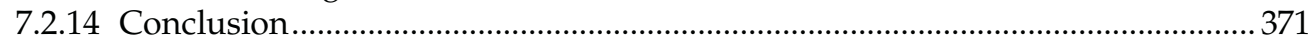

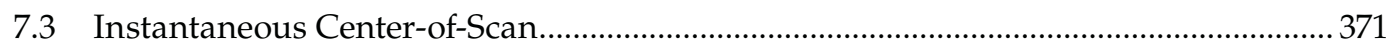

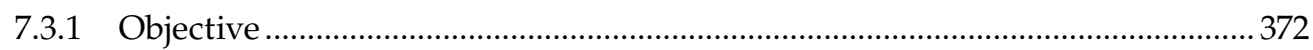

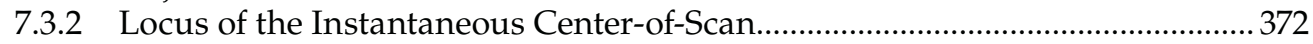

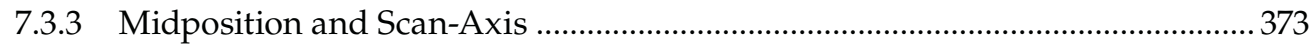

7.3.4 Derivation of the Instantaneous Center-of-Scan Coordinates...............................373

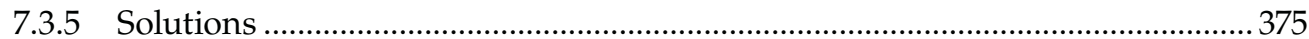

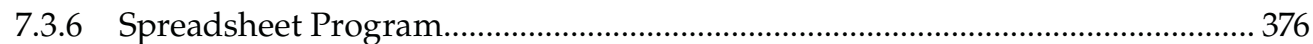

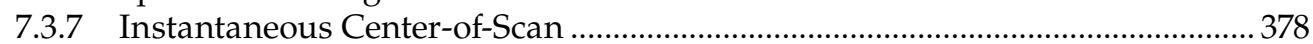

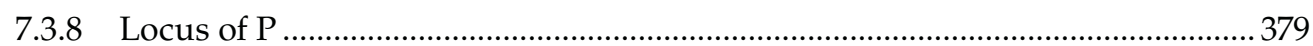

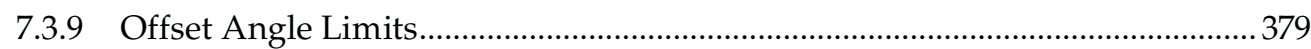

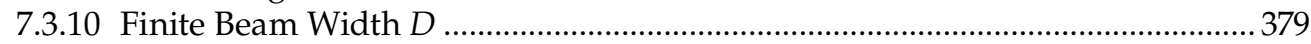

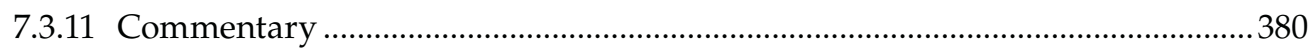

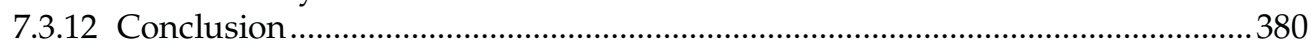


7.4 Stationary Ghost Images Outside the Image Format ..............................................380

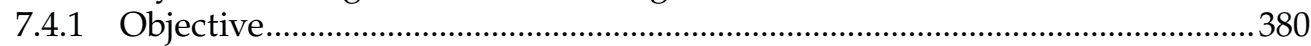

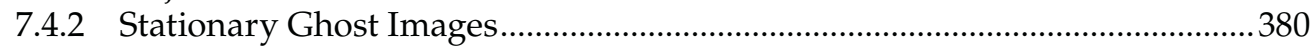

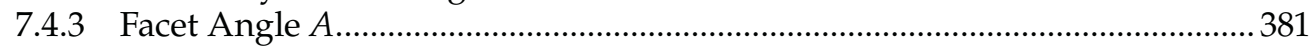

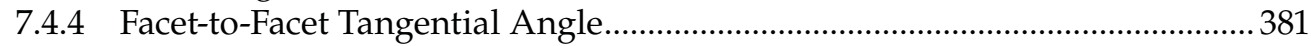

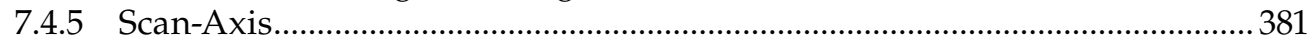

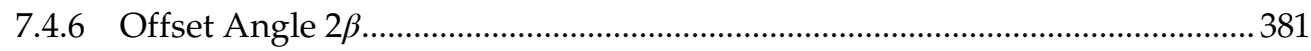

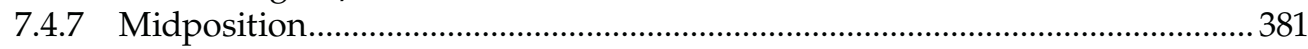

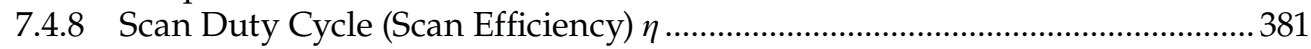

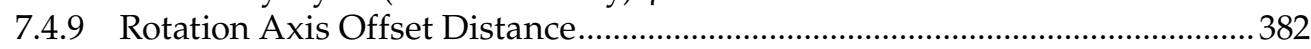

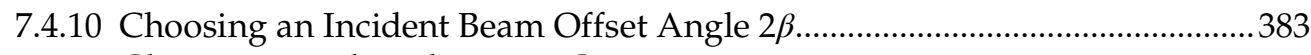

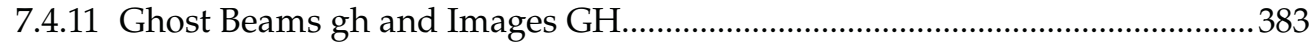

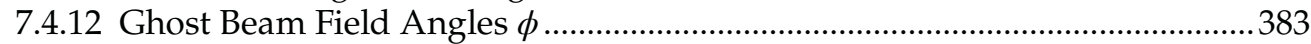

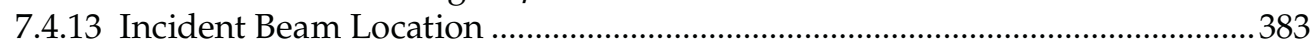

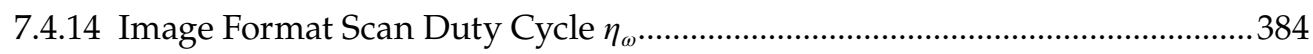

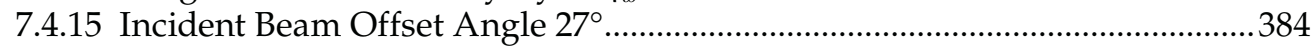

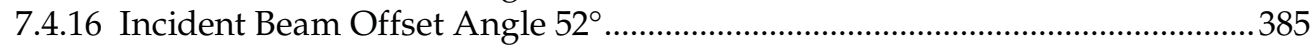

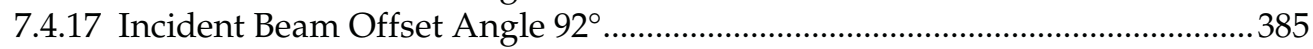

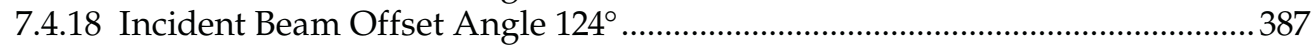

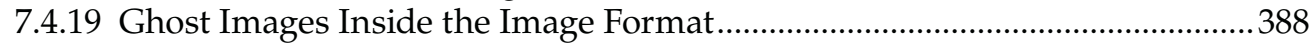

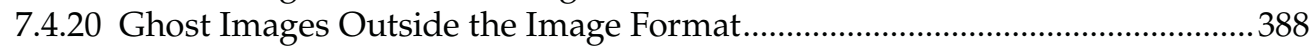

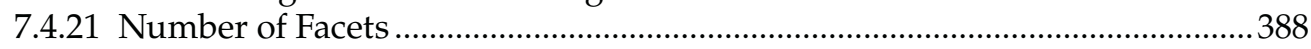

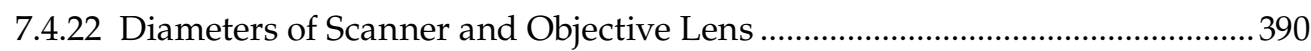

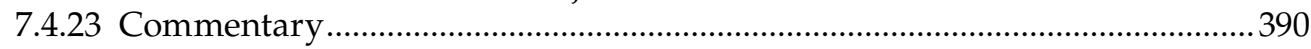

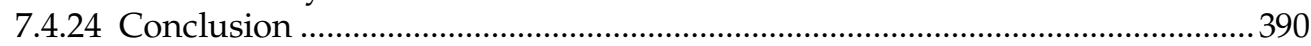

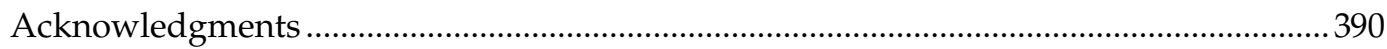

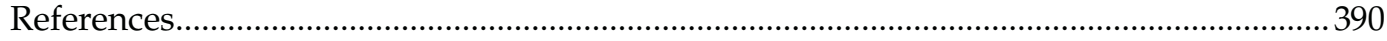

\subsection{INTRODUCTION}

Design equations for regular prismatic polygonal scanning systems have been analyzed and described by Kessler ${ }^{1}$ and Beiser. ${ }^{2,3}$ Beiser's analytical treatment is comprehensive in that the performance in terms of resolution is the key criterion used for the system designs and analyses. Henceforth, throughout this chapter, the term polygonal scanner shall infer a regular prismatic polygonal scanner.

The prime objective of this chapter is to provide a comprehensive visual understanding of the effects of changing the incident beam width (diameter) $D$, the incident beam offset angle $2 \beta$, which is the angle the incident beam is offset from the $x$-axis, and the number $N$ of mirror facets on a polygonal scanner without regard to performance in terms of resolution. Diagrams, equations, and coordinates bring to light these insights.

Cartesian rectilinear coordinate axes $\mathrm{O} x$ and $\mathrm{O} y$ are chosen for the equations of lines, loci, and the coordinates of significant points. The origin coincides with the axis of rotation $\mathrm{O}$ of the regular prismatic polygonal scanner. The $x$-axis $(\mathrm{O} x)$ is parallel to the optical axis of the objective lens.

There are three distinct topics associated with pre-objective polygonal scanning systems that are covered in this chapter by three separate sections, 7.2, 7.3, and 7.4. To assist a reader interested in only one of the topics certain definitions are repeated for continuity of a topic in a section so that the reader does not have to cross-refer back and forth to different sections. 
The topics are: equations and coordinates of a polygonal scanning system; instantaneous center-of-scan (ICS); and stationary ghost images outside the image format.

\subsubsection{Equations and Coordinates of a Polygonal Scanning System}

The midposition orientation of the polygonal scanner facets is such that the reflected collimated incident beam is parallel to the $x$-axis and defines the scan-axis, both of which are chosen to be parallel to the optical axis of the objective lens (Figure 7.1).

For a given incident beam offset angle $2 \beta$ of the collimated incident beam, the equations for the scan-axis, the incident beam, the mirror facet plane, and the objective lens optical axis scanner are expressed with respect to the rotation axis $\mathrm{O}$ of the polygonal scanner; likewise are the precise coordinates of significant points. ${ }^{4}$

\subsubsection{Instantaneous Center-of-Scan (ICS)}

Presented is the derivation of the parametric equations for the loci of the ICS for six-and twelve-facet prismatic polygonal scanners. ${ }^{5}$ Depicted are figures that show the changes in the loci characteristics for different incident beam offset angles $2 \beta$.

\subsubsection{Stationary Ghost Images Outside the Image Format}

Presented is a pictorial display of diagrams illustrating the permissible angular ranges of the incident beam offset angle $2 \beta$ to ensure that the ghost images lie outside the image format of the scanned field image format. ${ }^{6}$

\subsection{EQUATIONS AND COORDINATES OF A POLYGONAL SCANNING SYSTEM}

The origin of the Cartesian rectilinear coordinate axes can be chosen to be either at the rotation axis $\mathrm{O}$ of the polygonal scanner, or at the point of incidence $\mathrm{P}$ on a mirror facet;

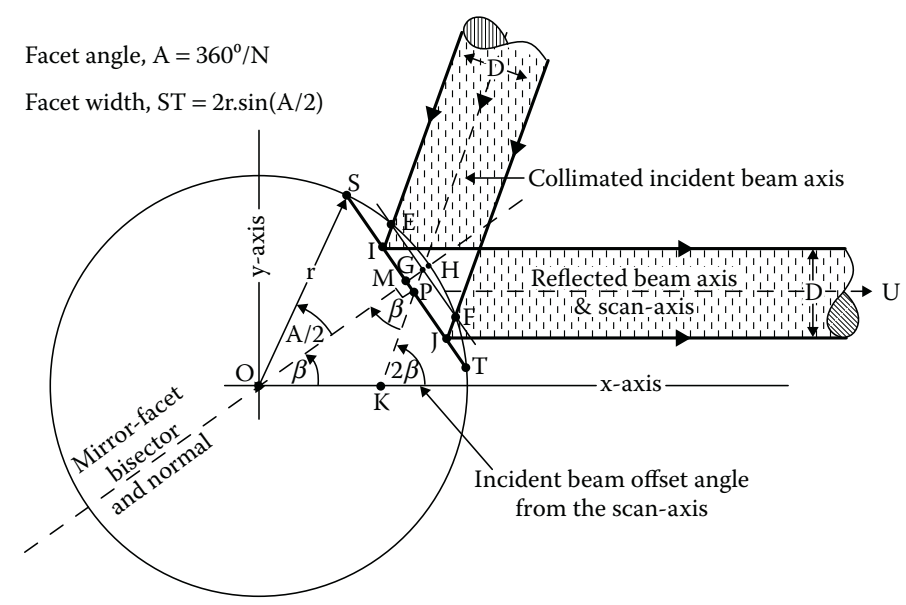

FIGURE 7.1

A single facet ST of a polygonal scanner oriented in the midposition. See Figure 7.2 for greater clarity around the point of incidence $P$. 
each approach has an advantage for giving insights. In this section the rotation axis $\mathrm{O}$ has been chosen to be the origin. ${ }^{4}$

Consider Figure 7.1. The diagram depicts a single facet ST of a regular prismatic polygonal scanner with $N$ facets and its circumscribed circle of radius $r$. The facet ST is oriented so that the collimated incident beam, which is offset at an angle $2 \beta$ from the $x$-axis, is reflected parallel to the $x$-axis. The beam has a finite width $D$ (see Section 7.2.5).

\subsubsection{Objective}

The goal is to present the precise coordinates of significant points, the distances between these points, and the equations of three axes (incident beam, scan, and objective lens) with respect to the rotation axis $\mathrm{O}$ of the polygonal scanner, thereby eliminating manual or computer-aided iterative techniques. Furthermore, it is to provide unexpected interesting insights into the limitations of the optomechanical design layouts of polygonal scanning systems.

\subsubsection{Midposition and Scan-Axis}

Shown in Figure 7.1 is a single facet ST of a polygonal scanner oriented in a midposition such that a collimated incident beam is reflected parallel to the $x$-axis. The reflected beam axis PU in this midposition defines the scan-axis.

\subsubsection{Mirror Facet Angle $A$}

From this midposition the reflected beam angularly scans symmetrically about the scanaxis through an angle of $\pm A$. Character symbol $A$ is the facet angle, which is the angle that the facet ST subtends at the rotation axis $\mathrm{O}$ of the polygonal scanner.

$$
A=\frac{360^{\circ}}{N}
$$

\subsubsection{Mirror Facet Width}

The tangential width of the facet ST of a regular prismatic polygonal scanner is:

$$
\mathrm{ST}=2 r \sin \left(\frac{A}{2}\right)=2 r \sin \left(\frac{180}{N}\right)
$$

\subsubsection{Beam Width (Diameter) $D$}

The Gaussian laser beam width represented by $D$ is the standard " $1 / e^{2}$ beam width" plus a margin of safety chosen by the system designer to minimize the imaged spot defects when a Gaussian beam is one-sidedly truncated by a facet edge as the polygonal scanner rotates. The margin of safety is inextricably linked to the desired scan duty cycle (scan efficiency) $\eta$ of the scanning system for a given $2 \beta, N$, and $r$. For a one-side truncation of the beam by the facet edges as the polygon rotates, optimally $D$ is $40 \%$ greater than the $1 / e^{2}$ beam width. ${ }^{2,3}$

The $1 / e^{2}$ beam width that is symbolized by $D_{1 / e^{2}}$ is the beam width (diameter) beyond which the residual laser beam power is $1 / e^{2}$ of the total power of a laser beam that has a 
Gaussian distribution. For a Gaussian distribution, it uniquely and directly also corresponds to the laser beam width (diameter) at which the beam irradiance has dropped to $1 / e^{2}$ of the axial peak laser beam irradiance (see Chapter 1 ).

In Figure 7.1, in which the polygonal scanner is in the midposition:

1. The boundaries of the incident beam width $D$ are designed to cut through the circumscribed circle at E and F such that the arcs SE and FT are equal. This ensures that the useful angular scan of the reflected beam is symmetrical about the scan-axis.

2. In Figures 7.1 and 7.2 the incident beam axis that has a finite width $D$ intersects the mirror-facet bisector $\mathrm{OMH}$ at the point $\mathrm{G}$, as it proceeds to impinge on the mirror-facet as the point P. G is aslo the midpoint of the chord EF. For an infinitesimal beam width $\mathrm{G}$ coincides with the point $\mathrm{H}$. As the beam increases $\mathrm{G}$ approaches $\mathrm{M}$ as does $\mathrm{P}$.

\subsubsection{Scan Duty Cycle (Scan Efficiency)}

The scan duty cycle $\eta$ of a polygonal scanner is the ratio of the useful scan angle during which the beam width $D$ is unvignetted by the edges of the facets, to the full scan angle $\pm A$ of a beam with an infinitesimal beam width. One assumes that the tangential width of the footprint of the incident beam is less than the tangential width of the facet, when the polygonal scanner is in its midposition (see Chapter 2 of this volume). Henceforth, widths refer to tangential widths.

It can be shown ${ }^{4}$ that

$$
h=1-\frac{\arcsin [D /(2 r \cos b)]}{180 / N}
$$

Knowing or selecting suitable values for $r, N, \beta$, and $D$ will determine $\eta$. Alternatively, choosing suitable values for $r, N, \beta$, and $\eta$ will determine the required incident beam width

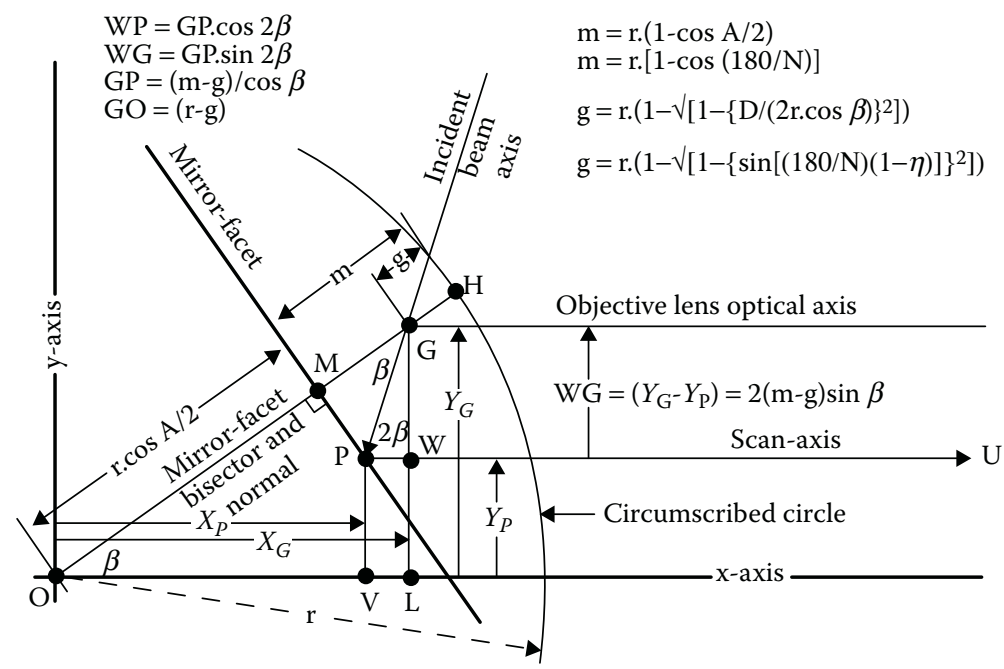

FIGURE 7.2

A geometrical diagram for determining the coordinates of $\mathrm{G}$ and $\mathrm{P}$, namely, $X_{\mathrm{G}}, Y_{\mathrm{G}}$ and $X_{P}, Y_{P}$. 
$D$ as follows. Transposing Equation 7.3 gives

$$
D=(2 r \cos b) \sin [(180 / N)(1-h)]
$$

More simply, if $W$ represents the tangential facet width, this expression approximates to 2,3

$$
D_{\text {approx }}=W(\cos b)(1-h)
$$

Dividing Equation 7.5 by Equation 7.4 gives

$$
D_{\text {approx }} / D=\frac{[\sin (180 / N)](1-h)}{\sin [(180 / N)(1-h)]}
$$

The closeness of $D_{\text {approx }}$ to $D$ is illustrated in Table 7.1.

Let the polygonal scanner be in its midposition, then:

1. If the incident beam has an infinitesimal width $(D=0)$, the circumscribed circle of the polygonal scanner intersects the axis of the beam at the point $\mathrm{H}$, which coincides with the top of the facet sag $\mathrm{MH}$. The scan duty cycle is $100 \%(\eta=1)$, ignoring the inevitable facet edge manufacturing roll-off (Figure 7.2).

2. If the incident beam has a finite width $D$ with a footprint that just covers the facet's tangential width $(D=2 r \sin (A / 2))$, the beam axis is directed at $\mathrm{M}$, at the base of the sag $\mathrm{MH}$. The scan duty cycle is $0 \%(\eta=0)$. Simultaneously, point $\mathrm{P}$ coincides with $\mathrm{M}$, which is the midpoint of the mirror-facet chord ST.

3. For all finite incident beam widths $D$ with a footprint width that is within the facet width $(D<2 r \sin (A / 2))$, the beam axis passes through the midpoint $G$ of the chord $\mathrm{EF}$, and which lies on the facet sag $\mathrm{MH}$, to impinge on the mirror-facet at the point P. The scan duty cycle is finite $(1>\eta>0)$ (Equation 7.3, Figures 7.1 and 7.2).

\subsubsection{Sag Dimensions}

It can be shown $\mathrm{n}^{4}$ and with reference to the geometry in Figure 7.2 that when sag $\mathrm{MH}=m$

$$
m=r\left[1-\cos \left(\frac{A}{2}\right)\right]=r\left[1-\cos \left(\frac{180}{N}\right)\right]
$$

\section{TABLE 7.1}

Ratio $\left[D_{\text {approx }} / D\right]$ for Scan Duty Cycle $\eta$ versus Number of Facets $N$

\begin{tabular}{llllll}
\hline & \multicolumn{5}{l}{$\boldsymbol{\eta}$} \\
\cline { 2 - 6 } & $\mathbf{0 . 0 0}$ & $\mathbf{0 . 2 5}$ & $\mathbf{0 . 5 0}$ & $\mathbf{0 . 7 5}$ & $\mathbf{1 . 0 0}$ \\
\hline$N=3$ & 1.00 & 0.92 & 0.87 & 0.84 & 0.83 \\
$N=6$ & 1.00 & 0.98 & 0.97 & 0.96 & 0.95 \\
$N=12$ & 1.00 & 0.99 & 0.99 & 0.99 & 0.97 \\
$N=18$ & 1.00 & 1.00 & 1.00 & 1.00 & 1.00 \\
$N=24$ & 1.00 & 1.00 & 1.00 & 1.00 & 1.00 \\
\hline
\end{tabular}


If the sag $\mathrm{GH}=g$, then in terms of $r, D$, and $\beta$

$$
g=r\left(1-\sqrt{1-\left[\frac{D}{(2 r \cos b}\right]^{2}}\right)
$$

Or from Equations 7.7 and 7.8

$$
\begin{aligned}
& (m-g)=r\left(-\cos \left(\frac{180}{N}\right)\right)+\sqrt{\left[1-\left(\frac{D}{2 r \cos b}\right)^{2}\right]} \\
& (r-g)=\sqrt{\left[1-\left(\frac{D}{2 r \cos b}\right)^{2}\right]}
\end{aligned}
$$

Likewise, in terms of $r, N$, and $\eta$

$$
\begin{gathered}
g=r\left(1-\sqrt{1-\left(\frac{D}{2 r \cos b}\right)^{2}}\right) \\
(m-g)=r\left(-\cos \left(\frac{180}{N}\right)\right)+\sqrt{\left[1-\left(\frac{D}{2 r \cos b}\right)^{2}\right]} \\
(r-g)=\sqrt{\left[1-\left\{\sin \left[\left(\frac{180}{N}\right)(1-h)\right]\right\}^{2}\right]}
\end{gathered}
$$

(see Figures 7.2 and 7.4).

\subsubsection{Coordinates of $G$}

From the geometry in Figure 7.2:

$$
X_{\mathrm{G}}=(r-g) \cos b
$$

and

$$
Y_{\mathrm{G}}=(r-g) \sin b
$$

Substituting for $(r-g)$ from Equation 7.10, and expressing $X_{\mathrm{G}}, Y_{\mathrm{G}}$ in terms of $\mathrm{r}, D$, and $\beta$ give

$$
X_{\mathrm{G}}=r\left(\sqrt{1-\left(\frac{D}{2 r \cos b}\right)^{2}}\right) \cos b
$$


and

$$
Y_{\mathrm{G}}=r\left(\sqrt{1-\left\{\frac{D}{2 r \cos b}\right\}^{2}}\right) \sin b
$$

Likewise, substituting for $(r-g)$ from Equation 7.10, and expressing $X_{\mathrm{G}}, Y_{\mathrm{G}}$ in terms of $r$, $N$, and $\eta$ give

$$
X_{\mathrm{G}}=r\left(\sqrt{1-\left\{\sin \left[\left(\frac{180}{N}\right)(1-h)\right]\right\}^{2}}\right) \cos b
$$

and

$$
Y_{\mathrm{G}}=r\left(\sqrt{\left[-\left\{\sin \left[\left(\frac{180}{N}\right)(1-h)\right]\right\}^{2}\right]}\right) \sin b
$$

\subsubsection{Coordinates of $\mathbf{P}$}

Again from the geometry in Figure 7.2:

$$
X_{\mathrm{P}}=X_{\mathrm{G}}-(m-g)\left[\frac{\cos 2 b}{\cos b}\right]
$$

and

$$
Y_{\mathrm{P}}=Y_{\mathrm{G}}-2(m-g) \sin b
$$

Substituting for $X_{\mathrm{G}}$ and $Y_{\mathrm{G}}$ from Equations 7.14 and 7.15 gives

$$
X_{\mathrm{P}}=(r-g) \cos b-(m-g)\left[\frac{\cos 2 b}{\cos b}\right]
$$

and

$$
Y_{\mathrm{P}}=(r-g) \sin (b-2)(m-g) \sin b
$$

By substituting for $(m-g)$ and $(r-g)$ from Equations 7.9 and 7.10 into Equations 7.22 and 7.23 one obtains the coordinates of $X_{\mathrm{P}}, Y_{\mathrm{P}}$ expressed in terms of $r, D$, and $\beta$.

$$
X_{\mathrm{P}}=\left(\frac{r}{\cos b}\right)\left[\cos \left(\frac{180}{N}\right) \cos 2 b+\sin 2 b \sqrt{\left(1-\left[\frac{D}{2 r \cos b}\right]^{2}\right)}\right]
$$


and

$$
Y_{\mathrm{P}}=(r \sin b)\left[2 \cos \left(\frac{180}{N}\right)-\sqrt{\left(1-\left[\frac{D}{(\cos b)}\right]^{2}\right)}\right]
$$

By substituting for $(m-g)$ and $(r-g)$ from Equations 7.12 and 7.13 into Equations 7.22 and 7.23 one obtains the coordinates of $X_{\mathrm{P}}, Y_{\mathrm{P}}$ expressed in terms $r, N$, and $\eta$.

$$
\begin{aligned}
& X_{\mathrm{P}}=\left(\frac{r}{\cos b}\right)\left[\cos \left(\frac{180}{N}\right) \cos 2 b+\sin 2 b \sqrt{\left(1-\left\{\sin \left[\left(\frac{180}{N}\right)(1-h)\right]\right\}^{2}\right)}\right] \\
& Y_{\mathrm{P}}=(r \sin b)\left[2 \cos \left(\frac{180}{N}\right)-\sqrt{\left(1-\left\{\sin \left[\left(\frac{180}{N}\right)(1-h)\right]\right\}^{2}\right)}\right]
\end{aligned}
$$

\subsubsection{Optical Axis of the Objective Lens}

The objective lens optical axis, which is parallel to both the $x$-axis and the scan-axis, is directed through the point $G$ to ensure that the scanning beam width $D$ scans symmetrically across the aperture of the objective lens (Figures 7.1, 7.2, and 7.3).

The separation between the objective lens optical axis and the scan-axis is given by

$$
\mathrm{WG}=2(m-g) \sin b
$$

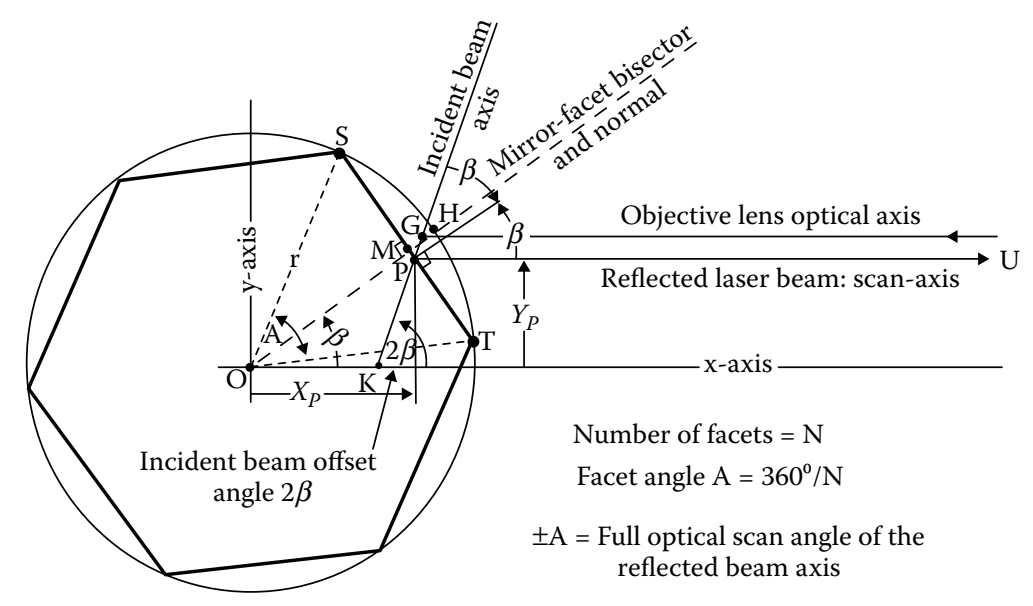

FIGURE 7.3

The six facets of the hexagonal scanner head oriented in the midposition at which the reflected incident beam axis lies along the scan-axis. The boundaries of the beam, which has a width $D$, are omitted to avoid overcrowding the diagram. 
If the incident beam has an infinitesimal width, G coincides with $\mathrm{H}(g=0)$, and the separation WG between the objective lens optical axis and the scan-axis is a maximum

$$
\mathrm{WG}_{\max }=2 m(\sin b)
$$

Substituting for $m$ from Equation 7.7 leads to

$$
\mathrm{WG}_{\max }=2 r\left[1-\cos \left(\frac{180}{N}\right)\right] \sin b
$$

As $G$ and P simultaneously approach $M$ the objective lens optical axis and the scan-axis move toward each other at the same rate in the $y$-axis direction until they both coincide at $\mathrm{M}$.

When the incident beam has a finite width $D$ and the beam width of the footprint just covers the facet chord ST, G and P coincide with M, $m=g,(m-g)=0$.

Substituting $(m-g)=0$ into Equation 7.28 gives

$$
\mathrm{WG}_{\min }=0
$$

Thus, the objective lens axis is coincident with the scan-axis.

\subsubsection{Equations}

Except for the incident beam, the scan-axis and objective lens optical axis are parallel to the $x$-axis and, therefore, have equations independent of $x$.

\subsubsection{Scan-Axis PU}

The equation to the scan-axis corresponds to $Y_{\mathrm{P}}$, given in Equation 7.23, namely

$$
Y_{\mathrm{P}}=(r-g) \sin b-2(m-g) \sin b
$$

See Equations 7.25 and 7.27.

In a reverse sense $Y_{P}$ also represents the offset distance of the rotation axis $O$ from the scan-axis PU for a given offset angle $\beta$ of the incident beam (see Sections 7.2.12 and 7.4.9).

\subsubsection{Objective Lens Optical Axis}

The equation to the objective lens optical axis corresponds to $Y_{\mathrm{G}}$ given in Equation 7.15, namely

$$
Y_{\mathrm{G}}=(r-g) \sin b
$$

From Equation 7.17, expressing $Y_{\mathrm{G}}$ in terms of $r, D$, and $\beta$ gives

$$
Y_{\mathrm{G}}=r\left(\sqrt{1-\left[\frac{D}{(2 r \cos b)}\right]^{2}}\right) \sin b
$$


From Equation 7.19, expressing $Y_{\mathrm{G}}$ in terms of $r, N$ and $\eta$ gives

$$
Y_{\mathrm{G}}=r\left(\sqrt{\left[1-\left\{\sin \left[\left(\frac{180}{N}\right)(1-h)\right]^{2}\right\}\right]}\right) \sin b
$$

\subsubsection{Incident Beam Axis Through GP}

$$
y=(\tan 2 b) x-(r-g)[(\tan 2 b)(\cos b)+\sin b]
$$

where from Equation $7.10(r-g)$ is expressed in terms of $r, D$, and $\beta$, namely

$$
(r-g)=\sqrt{\left[1-\left(\frac{D}{2 r \cos b}\right)^{2}\right]}
$$

Alternatively, where from Equation $7.13(r-g)$ is expressed in terms of $r, N$, and $\eta$, namely

$$
(r-g)=\left[\sqrt{1-\left\{\sin \left[\left(\frac{180}{N}\right)(1-h)\right]\right\}^{2}}\right]
$$

\subsubsection{Mirror Facet Bisector and Normal}

The linear equation to the bisector of the mirror facet and normal has a slope of $\tan \beta$ with no intercept and passes through the rotation axis $\mathrm{O}$, which is the origin of the coordinate system. Thus

$$
y=x(\tan b)
$$

\subsubsection{Insights from an Alternative Analytical Approach}

An alternative perspective for the analysis is to set the Cartesian rectilinear coordinate axes to be $\mathrm{P} x$ and $\mathrm{P} y$ with the origin at the point of incidence $\mathrm{P}$ on the facet for when the polygonal scanner is in a midposition (Figure 7.4). In this approach the scan-axis is collinear with the abscissa, the $x$-axis $(\mathrm{P} x)$, while the ordinate is the $y$-axis $(\mathrm{P} y)$. See the first paragraph at the beginning of Section 7.2.

The immediate advantage is that equations for the scan-axis, the incident beam axis, and the facet plane all pass through the origin $\mathrm{P}$. The goal of this second approach is to determine the coordinates $\left(X_{\mathrm{O}}, Y_{\mathrm{O}}\right)$ of the rotation axis $\mathrm{O}$ of the polygonal scanner with respect to the point of incidence $P$ and the scan-axis $P x$.

The approach presents a diagrammatic visualization of the existence of a finite areal zone, with respect to the fixed point $\mathrm{P}$, of a set of loci for the rotation axis $\mathrm{O}_{N}$ of a polygonal scanner that results from changes in the number $N$ of facets $(3 \leq N<\infty)$, the laser beam width $D$, and the scan duty cycle $\eta,(0 \leq \eta \leq 1)$, for a given circumscribed circle of radius $r$ of the polygonal scanner (Figure 7.4). ${ }^{4}$ 


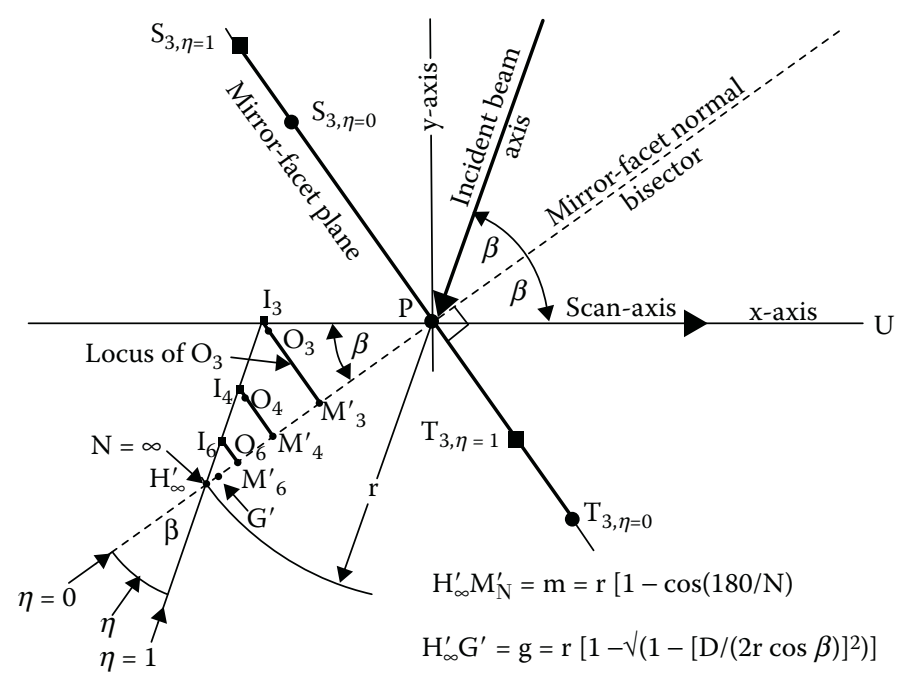

\section{FIGURE 7.4}

Displayed are the loci of the rotation axes $\mathrm{O}_{N}$ relative to the point of incidence $\mathrm{P}$ on a mirror-facet plane of a polygonal scanner oriented in a midposition. $\mathrm{I}_{3}$ is the rotation axis $\mathrm{O}_{3}$ for a three mirror-faceted polygon $\mathrm{N}=3$ for an infinitesimal beam width, such that $\eta=1$. As the beam width increases $\mathrm{O}_{3}$ moves along $\mathrm{I}_{3} \mathrm{M}_{3}^{\prime}$ toward $\mathrm{M}_{3}^{\prime}$ where $\eta=0$. Likewise for $\mathrm{I}_{4}$ and $\mathrm{I}_{6} ;$ as $\mathrm{O}_{4}$ and $\mathrm{O}_{6}$ move along $\mathrm{I}_{4} \mathrm{M}_{4}^{\prime}$ and $\mathrm{I}_{6} \mathrm{M}_{6}^{\prime}$, respectively.

All these coordinates and equations can be obtained from those already given in Sections 7.2.8 to 7.2.11 by the transformation of the origin at $\mathrm{O}$ to an origin at $\mathrm{P}$.

\subsubsection{Features of Figure 7.4}

In Figure 7.4, the loci for $N=5$ and for $N>6$ are omitted to avoid overcrowding the diagram. Certain character symbols are primed because of a direct, but not obvious, relationship to those corresponding unprimed symbols in Figure 7.2.

The set of loci for the position of the rotation axis $\mathrm{O}_{N}$ are confined to the series of parallel base lines $\mathrm{I}_{N} \mathrm{M}_{N}$ of a nest of right triangles $\mathrm{H}_{\infty}^{\prime} \mathrm{M}_{N}^{\prime} \mathrm{I}_{N}$ within the triangle $\mathrm{H}_{\infty}^{\prime} \mathrm{M}_{3}^{\prime} \mathrm{I}_{3}$. These base lines are parallel to the facet plane ST (Table 7.2).

$$
\mathrm{I}_{N} \mathrm{M}_{N}=m \tan b=r\left[1-\cos \left(\frac{180}{N}\right)\right] \tan b
$$

The base lines $\mathrm{I}_{n} \mathrm{M}_{n}$ are spaced at ever diminishing distances toward the apex $\mathrm{H}_{\infty}^{\prime}$ of the triangle as the number $N$ of facets increases. The spacing between every sixth base line is given by

$$
\left[m_{N}-m_{N+6}\right]=r\left[\cos \left(\frac{180}{N+6}\right)-\cos \left(\frac{180}{N}\right)\right]
$$

Simultaneously at a lesser rate, the facet widths $S_{N} T_{N}$ shorten as the number $N$ of facets increases:

$$
\mathrm{S}_{N} \mathrm{~T}_{N}=2 r \sin \left(\frac{180}{N}\right)
$$


TABLE 7.2

Facet Width $W_{N}$ and Locus Length $\mathrm{I}_{N} \mathrm{M}_{N}$ versus Number of Facets $N$ for $\mathrm{r}=50 \mathrm{~mm}$

\begin{tabular}{lcccc}
\hline & \multicolumn{4}{c}{$\boldsymbol{N}$} \\
\cline { 2 - 5 } & $\mathbf{6}$ & $\mathbf{1 2}$ & $\mathbf{1 8}$ & $\mathbf{2 4}$ \\
\hline$W_{N}=\mathrm{S}_{N} \mathrm{~T}_{N}(\mathrm{~mm})$ & 50.00 & 25.9 & 17.4 & 13.1 \\
$\mathrm{I}_{N} \mathrm{M}_{N}(\mathrm{~mm})$ & 3.87 & 0.46 & 0.13 & 0.06 \\
{$\left[\mathrm{~S}_{N} \mathrm{~T}_{N}\right]\left[\mathrm{I}_{N} \mathrm{M}_{N}\right]$} & 12.9 & 56.7 & 130 & 232 \\
{$\left[m_{N} \otimes m_{N+6}\right](\mathrm{mm})$} & 5.00 & 0.94 & 0.33 & 0.15 \\
\hline
\end{tabular}

From Equations 7.42 and 7.40 the ratio of the facet width $S_{N} T_{N}$ to length of the locus $I_{N} M_{N}$ is expressed by

$$
\frac{\mathrm{S}_{N} \mathrm{~T}_{N}}{\mathrm{I}_{N} \mathrm{M}_{N}}=2[\operatorname{cotan}(90 / N)]^{2}
$$

for which the incident beam offset angle $2 \beta=+A$.

The position of the rotation axis on a locus $\mathrm{I}_{N} \mathrm{M}_{N}$ depends on the scan duty cycle $(0<\eta<1)$. A fan of straight lines emanating from $\mathrm{H}_{\infty}^{\prime}$ toward $\mathrm{I}_{N} \mathrm{M}_{N}$ represents a set of values for constant scan duty cycle $\eta$. The rotation axis $\mathrm{O}_{n}$ lies at the intersection of one of these fan lines of constant $\eta$ with a base line $\mathrm{I}_{N} \mathrm{M}_{N}$.

A set of straight lines parallel to $\mathrm{H}_{\infty}^{\prime} \mathrm{I}_{3}$ represents a set of values for constant beam width $D$. Similarly, the rotation axis $\mathrm{O}_{N}$ lies at the intersection of one of these parallel lines of constant $D$ with a base line $\mathrm{I}_{N} \mathrm{M}_{N}$. The rotation axes $\mathrm{O}_{N}$ may not lie beyond $\mathrm{M}_{N}$ where the incident beam width footprint matches the facet width.

All facet widths $S_{N} T_{N}$ lie between the points $S_{3, h=1}$ and $T_{3, h=0}$ according to the values of $N$ and $\eta$. The positional range of facet $S_{N} T_{N}$ directly corresponds to the range of the locus $\mathrm{O}_{N}$, that is, the length of the baseline $\mathrm{I}_{N} \mathrm{M}_{N}$. Uniquely, the rotation axis $\mathrm{O}$ lies on the scan-axis only when $N=3$ and $D=0$, that is, an incident beam of infinitesimal width.

\subsubsection{Conclusion}

The visualization of the effects of changing the controlling parameters $N, \beta, D, \eta$, and $r$ of an optical scanning system helps in its design, while, in particular, the explicit coordinates and equations eliminate manual or computer-aided iterative techniques.

\subsection{INSTANTANEOUS CENTER-OF-SCAN}

Reflective scanning devices, resonant, galvanometric, and polygonal, have plane mirrors that oscillate or rotate about an axis. The rotation of the reflecting mirror deflects an incident light beam. When (1) the axis of rotation $\mathrm{O}$ is coincident with the mirror surface, and (2) the incident beam is directed at the axis of rotation, the ICS is a single stationary point on, and at, the axis of rotation $\mathrm{O}$ for all angular positions of the mirror. These 
two conditions are difficult to achieve and are rarely met; as a result, the ICS moves with respect to the rotation axis $\mathrm{O}$, and, therefore, is a locus (Figure 7.5). ${ }^{5}$

\subsubsection{Objective}

This section explores and illustrates the characteristic form of the ICS locus for polygonal scanners with respect to the incident beam offset angle $2 \beta$; that is, the angle between the incident beam and the scan-axis. The analysis, study, and depiction of the ICS loci for several incident beam offset angles for regular prismatic polygonal scanners of six and 12 facets give a visual appreciation of the asymmetry in the optical path lengths of the deflected beam as it sweeps through the full scan angle $\pm A$. These characteristics provide interesting insights for consideration when undertaking the design of a polygonal scanning system.

\subsubsection{Locus of the Instantaneous Center-of-Scan}

In Figure 7.5a, the center-of-scan of the reflected beam is a stationary point on the rotation axis $\mathrm{O}$. This is because two conditions are met: (1) the axis of rotation lies in the reflecting surface of a mirror (reflector); and (2) the incident beam is directed at the axis of rotation $\mathrm{O}$ of the mirror.

In Figure 7.5b, the center-of-scan is not a stationary point, because, although the axis of rotation lies in the reflecting surface of a mirror, the incident beam is directed to one side of the rotation axis $\mathrm{O}$. However, there is an ICS at point $\mathrm{C} \equiv(\alpha, \gamma)$ that has a locus.

(a)

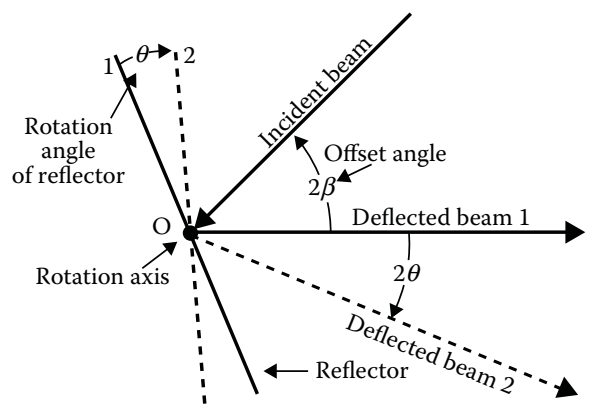

(c)

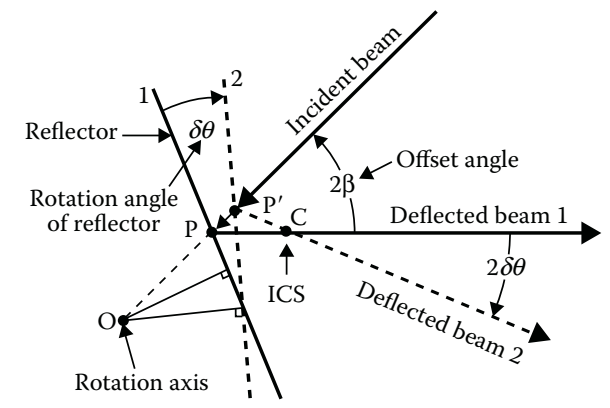

(b)

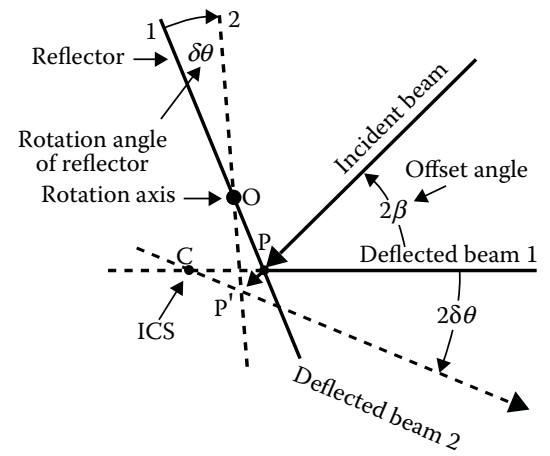

\section{FIGURE 7.5}

(a) The axis of rotation lies in the reflecting surface of a mirror (reflector) and the incident beam is directed at the axis of rotation $\mathrm{O}$ of the mirror, (b) The axis of rotation lies in the reflecting surface of a mirror but the incident beam is directed to one side of rotation axis $\mathrm{O}$. (c) The incident beam is directed at the axis of rotation but the rotation axis $\mathrm{O}$ is displaced from the reflecting surface of the mirror. 
Again, in Figure 7.5c, the center-of-scan is not a stationary point, because, although the incident beam is directed at the axis of rotation, the rotation axis $\mathrm{O}$ is displaced from the reflecting surface of the mirror. Similarly, there is an ICS at point $\mathrm{C} \equiv(\alpha, \gamma)$ that has a locus.

\subsubsection{Midposition and Scan-axis}

Figure 7.6 depicts a cross section of a hexagonal polygonal scanner set in a midposition with an incident beam offset angle $2 \beta$ of $70^{\circ}$. The midposition is defined by two requirements: (1) the polygonal scanner is oriented such that the reflected incident beam from one of the facets is parallel to the $x$-axis (this reflected incident beam defines the scan-axis); and (2) the rotation axis $\mathrm{O}$ is offset from the scan-axis to a position such that, as the polygonal scanner rotates, the reflected incident beam angularly scans symmetrically $\pm A$ about the scan-axis.

\subsubsection{Derivation of the Instantaneous Center-of-Scan Coordinates}

Consider a regular prismatic polygonal scanner with $N$ facets and a circumscribed circle of radius $r$ (Figure 7.6). Cartesian rectilinear coordinate axes $\mathrm{O} x$ and $\mathrm{O} y$ are chosen for the equations of lines, loci, and the coordinates of significant points. The origin $\mathrm{O}$ coincides with the axis of rotation of the polygonal scanner. The $x$-axis $(\mathrm{O} x)$ is parallel to the optical axis of the objective lens. The facet angle $A$, that is, the angle that the facet subtends at the axis of rotation $\mathrm{O}$, is given by $360 \% \mathrm{~N}$. For simplicity it is assumed that the beam width (diameter) is infinitesimal, such that a single ray represents the incident beam. ICS loci for finite beam widths $D$ are considered in Section 7.3.10.

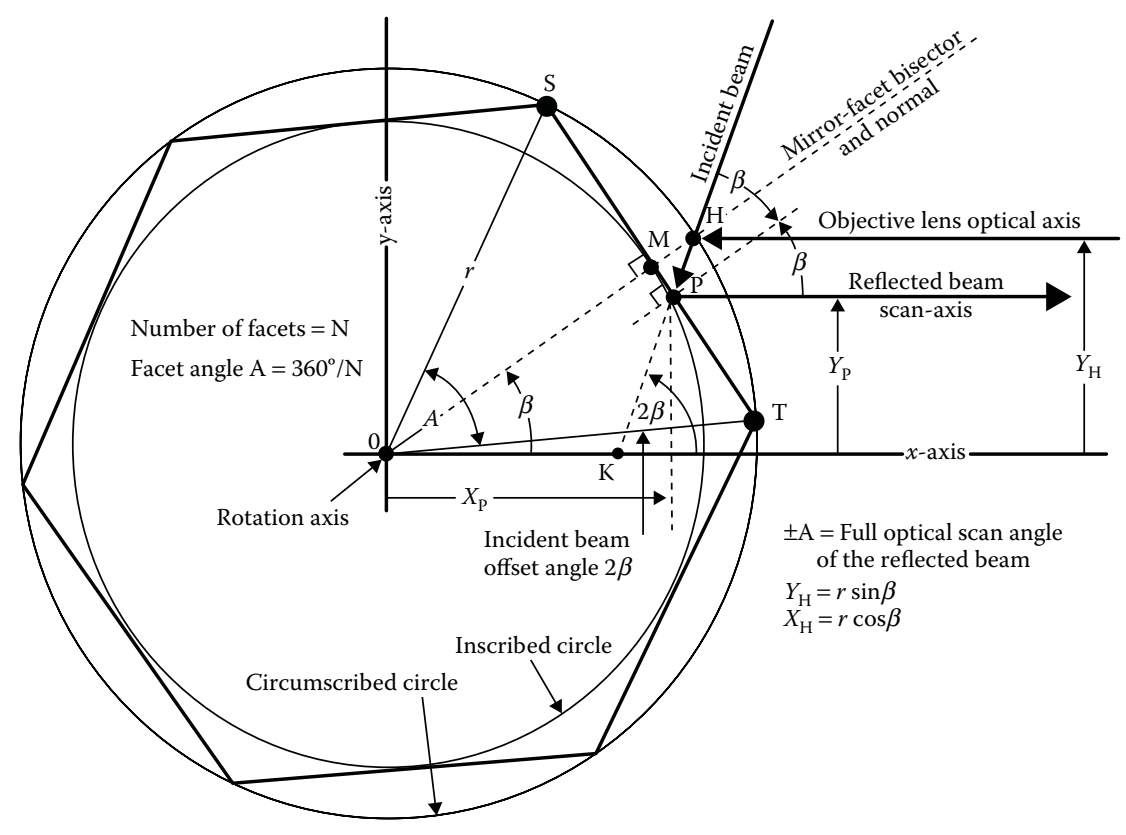

FIGURE 7.6

A scaled cross section of a six-facet polygonal scanner in the midposition from which the incident beam at an offset angle of $2 \beta$ is reflected parallel to both the objective lens optical axis and the $x$-axis. This reflected incident beam defines the scan-axis. 
Consider now an incident beam directed at the facet of a polygonal scanner in a midposition at an offset angle $2 \beta\left(70^{\circ}\right)$ (Figure 7.6). Point $\mathrm{H}$ on the incident beam is where the circumscribed circle of the polygonal scanner and a facet bisector OM scanner intersects the incident beam.

Figure 7.7 depicts the position of one facet of the polygon after it has been rotated counterclockwise through an angle $\theta$, and the resultant position and direction of the reflected incident beam that has been deflected through an angle $2 \theta$.

The linear equation of the reflected beam passes through the ICS coordinates $(\alpha, \gamma)$ and is represented by

$$
(y-g)=[\tan (2 q)](x-a)
$$

The incident beam linear equation expressed in the intercept form is

$$
\frac{x}{(r / 2) / \cos b}+\frac{y}{-(r / 2)[\tan (2 b)] / \cos b}=1
$$

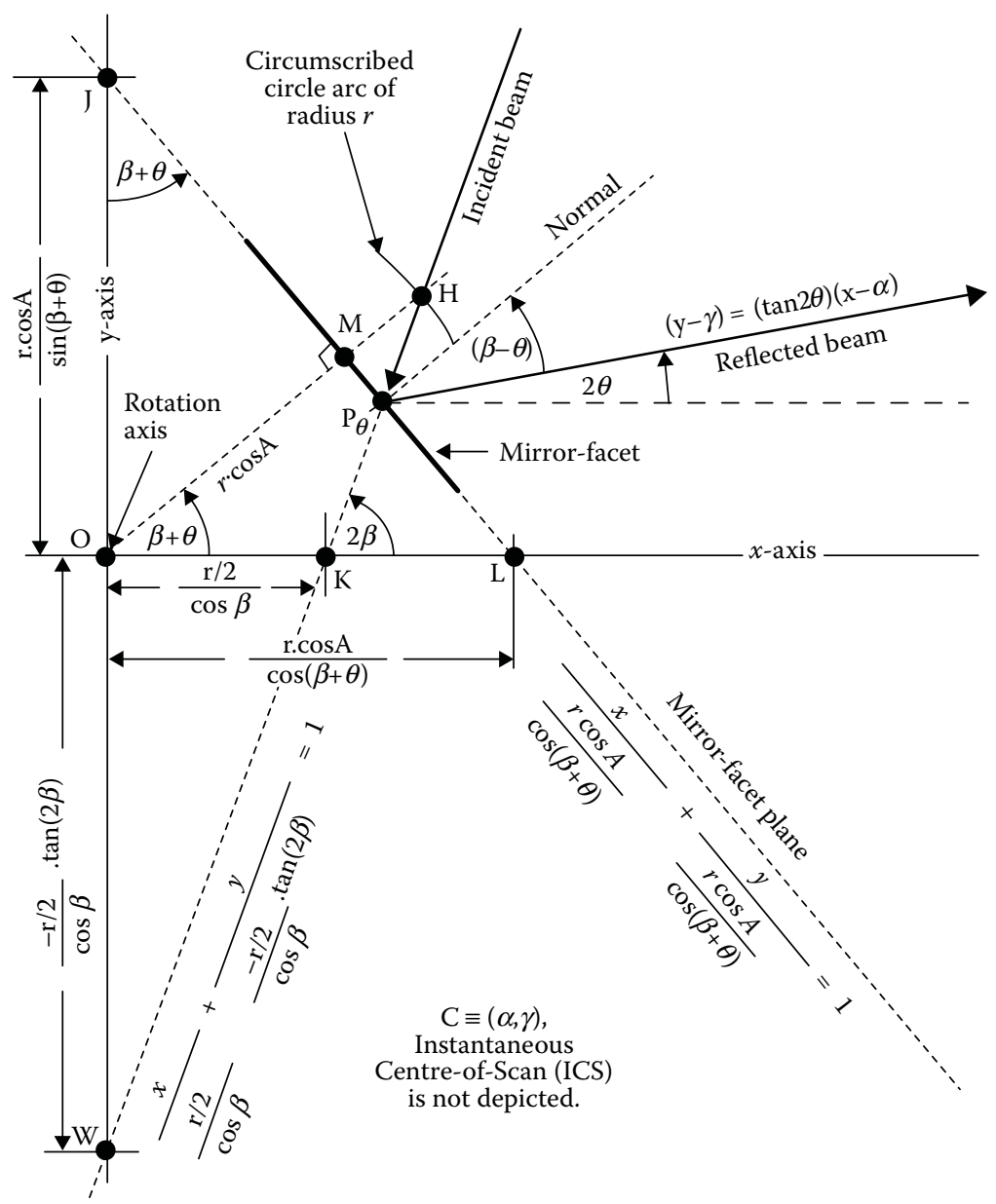

\section{FIGURE 7.7}

A trigonometrical diagram depicts the three key analytical equations of one facet of the polygonal scanner illustrated in Figure 7.6 in which the diagram has been rotated counterclockwise through an angle $\theta$. 
The linear equation for the line of intersection of the facet plane and the plane of incidence expressed in the intercept form is

$$
\frac{x}{r \cos A / \cos (b+q)}+\frac{y}{r \cos A / \sin (b+q)}=1
$$

From the three Equations 7.44, 7.45, and 7.46 the coordinates of $\alpha$ and $\gamma$ may be determined. The technique is to differentiate Equations 7.44 and 7.45 with respect to $\theta$ remembering that $\alpha$ and $\gamma$ are not variables, but constants at any instant.

Thus the derivative of Equation 7.44 is

$$
(x-a)=\frac{(\cos 2 q)^{2}\left(y^{\prime}-x^{\prime} \tan 2 q\right)}{2}
$$

And the derivative of Equation 7.45 is

$$
y^{\prime}=x^{\prime} \tan 2 b
$$

\subsubsection{Solutions}

Solving for $(x-\alpha)$ and $(y-\gamma)$ : eliminating $y^{\prime}$ between Equations 7.47 and 7.48 gives

$$
(x-a)=\frac{x^{\prime}(\cos 2 q)^{2}(\tan 2 b-\tan 2 q)}{2}
$$

Substituting for $(x-\alpha)$ from Equation 7.44 into Equation 7.49 leads to

$$
(y-g)=\frac{x^{\prime}(\sin 2 q)(\cos 2 q)(\tan 2 b-\tan 2 q)}{2}
$$

Inspection of Equations 7.49 and 7.50 shows the need to solve and substitute for $x$ and $y$, and $x^{\prime}$ with expressions containing only $r, A, \beta$, and $\theta$.

Solving for $x$ and $y$, and $x^{\prime}$

Note that simultaneous Equations 7.45 and 7.46 do not contain the ICS coordinates $\alpha$ and $\gamma$, thus solving for $x$ and $y$ gives the following parametric equations in terms of $r, \theta, A$, and $\beta$ :

$$
x=\frac{r[\cos A / \tan 2 b+\sin (b+q) / 2 \cos b]}{[\sin (b+q)+\cos (b+q) / \tan 2 b]}
$$

Likewise

$$
y=\frac{r[\cos A-\sin (b+q) / 2 \cos b]}{[\sin (b+q)+\cos (b+q) / \tan 2 b]}
$$

Equations 7.51 and 7.52 also represent the locus of the point of incidence $\mathrm{P},\left(X_{\mathrm{P}_{q}}, Y_{\mathrm{P}_{q}}\right)$, as $\theta$ varies and as the reflected incident beam scans. P inherently lies along the segment $\mathrm{HP}$ of the incident beam (Figures 7.6 and 7.7). 


\subsubsection{Spreadsheet Program}

The derivative $x^{\prime}$ is obtained by differentiating Equation 7.51 with respect to $\theta$. An explicit expression is possible but unnecessary when using a computer spreadsheet program. Tabulating data against $\theta$, obtained by using a spreadsheet program, are the values of $(x-\alpha)$ and $(y-\gamma)$ from Equations 7.49 and 7.50; the values of $x$ and $y$ from Equations 7.51 and 7.52, and the values of the derivative $x^{\prime}$; thence the coordinates $\alpha$ and $\gamma$ are deduced and plotted (Figures 7.8 through 7.12).

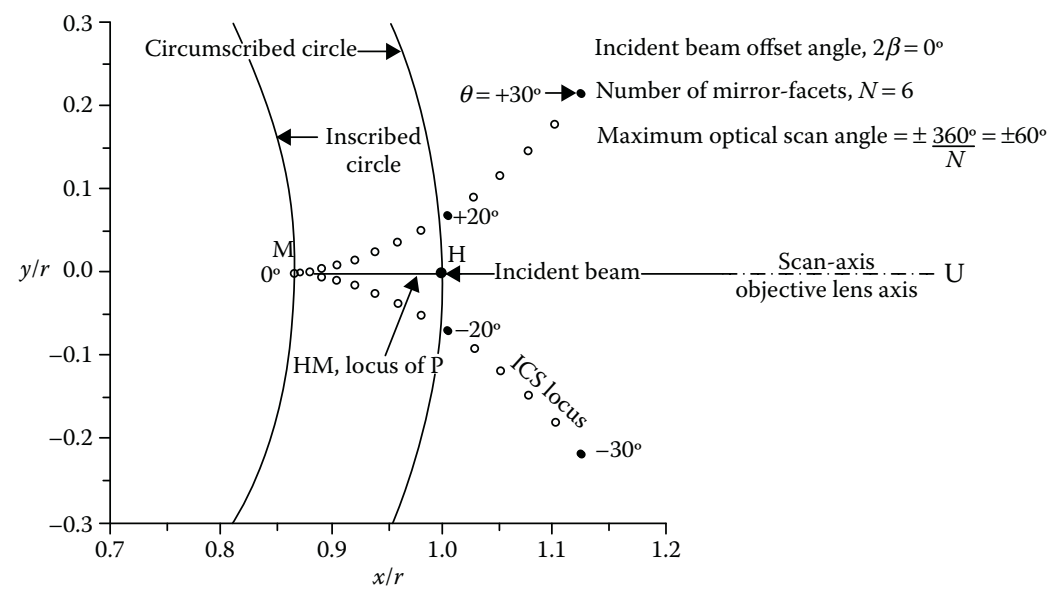

FIGURE 7.8

The ICS locus for an incident beam at an offset angle $2 \beta$ of $0^{\circ}$ for a six-facet polygonal scanner.

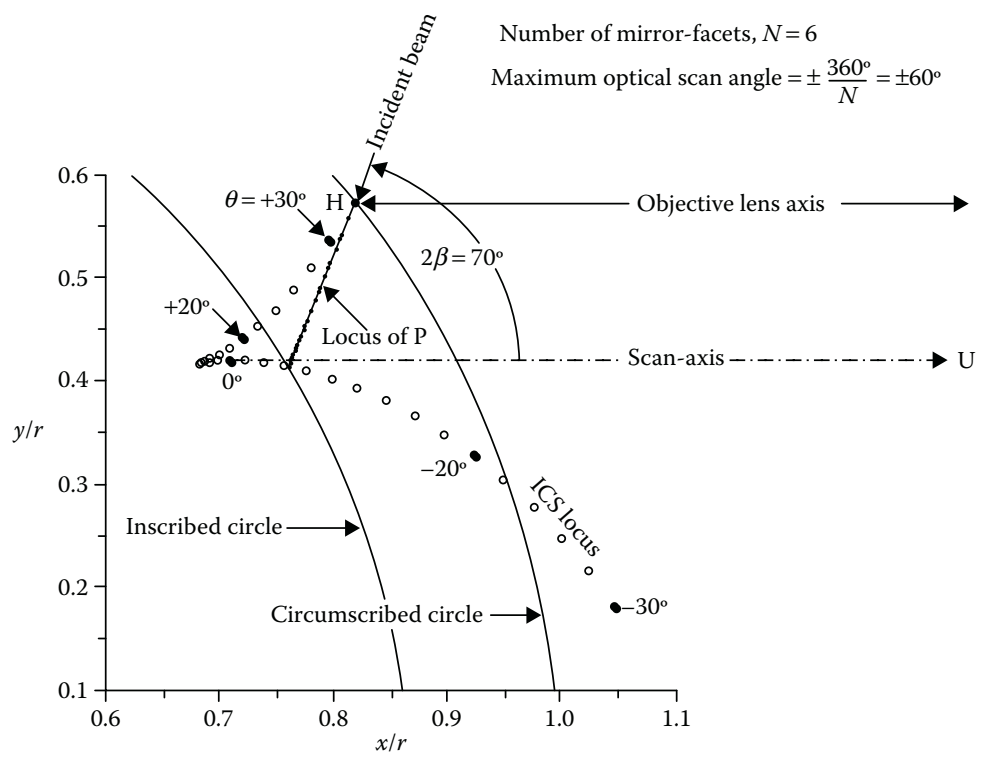

FIGURE 7.9

The ICS locus for an acute incident beam at an offset angle $2 \beta$ of $70^{\circ}$ for a six-facet polygonal scanner. 


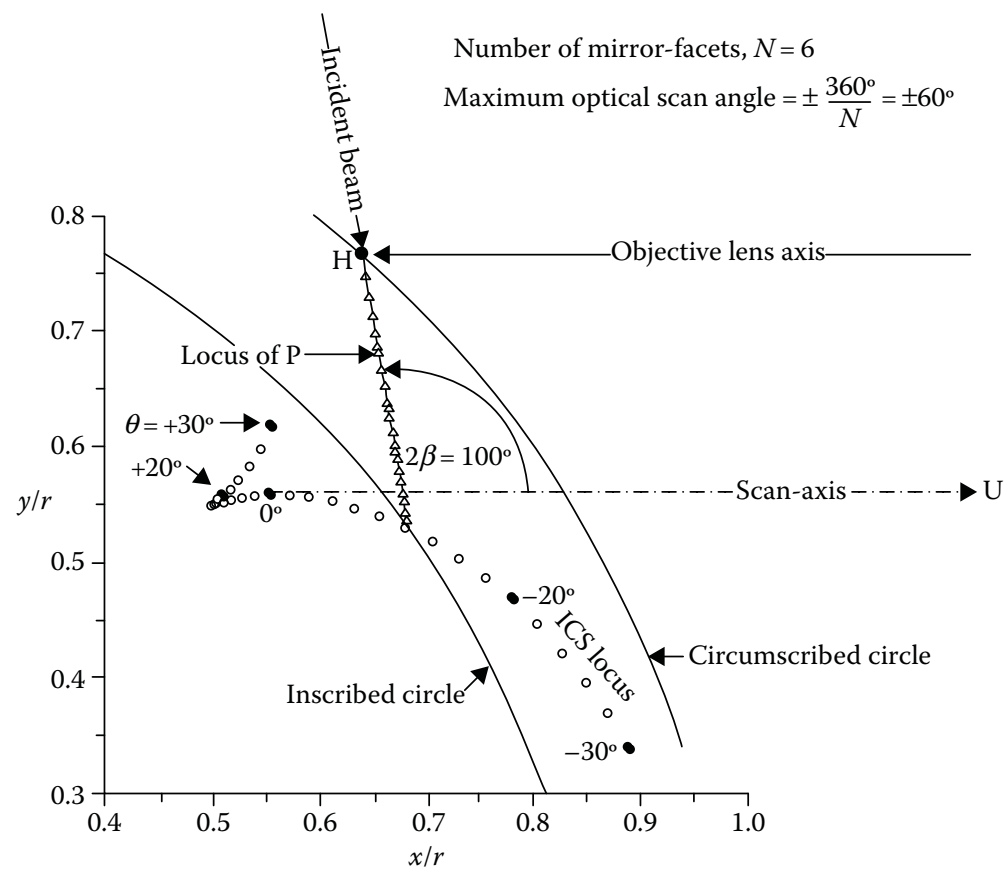

FIGURE 7.10

The ICS locus for an obtuse incident beam at an offset angle $2 \beta$ of $100^{\circ}$ for a six-facet polygonal scanner.

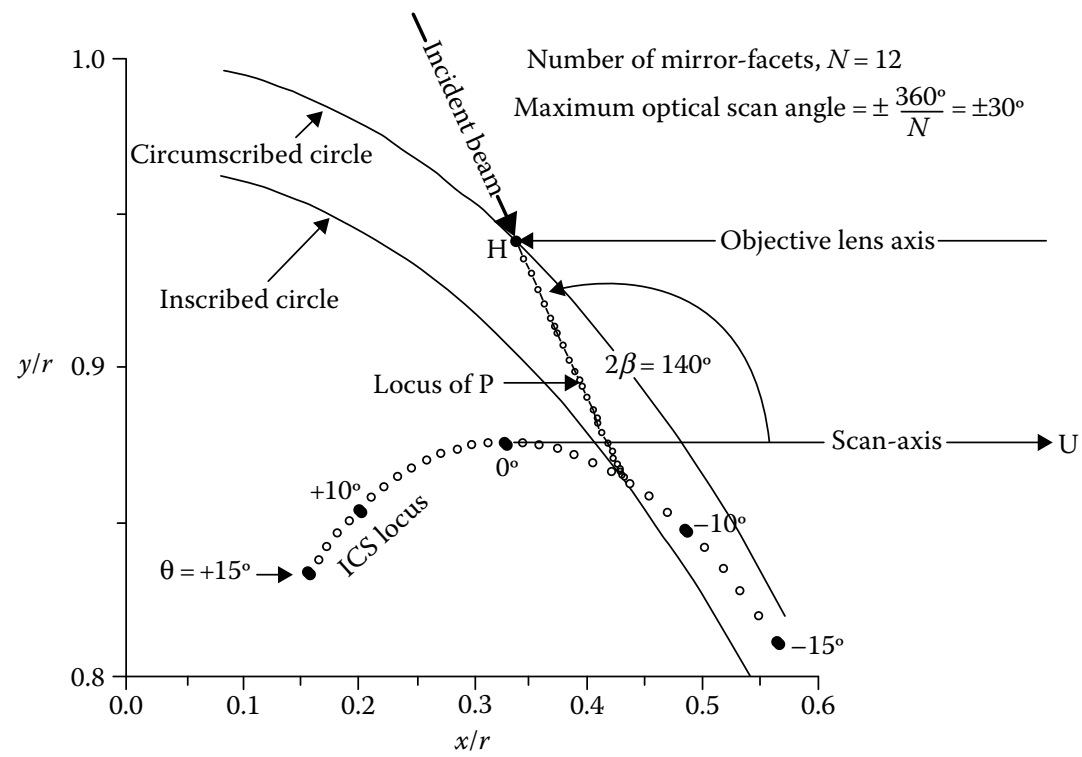

FIGURE 7.11

The ICS locus for an obtuse incident beam at an offset angle $2 \beta$ of $140^{\circ}$ for a 12 -facet polygonal scanner. A tangent to the ICS locus represents the position and direction of the reflected incident beam. 


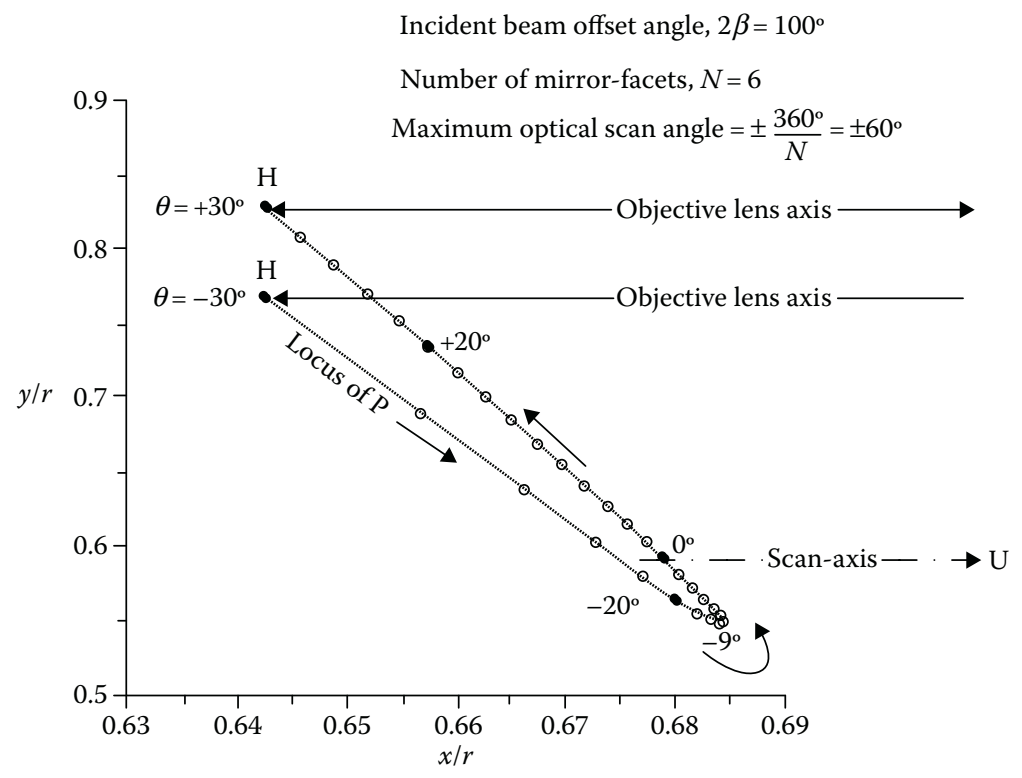

FIGURE 7.12

The locus of $\mathrm{P}$ for an incident beam at an offset angle $2 \beta$ of $100^{\circ}$ of Figure 7.10 . For visibility and clarity the ordinate data and scale of the abscissa have been adjusted.

\subsubsection{Instantaneous Center-of-Scan}

Figures 7.8 to 7.11 display the ICS loci for four incident beam $\beta$ offset angles, namely, $0^{\circ}, 70^{\circ}$, $100^{\circ}$, and $140^{\circ}$. The data plots on the ICS loci correspond to the mechanical rotation angle $\theta$ of the polygonal scanner at two-degree intervals from its midposition.

It should be noted that a tangent at any point on the ICS locus is the position and direction of the reflected incident beam for a rotation angle $\theta$. When the facet edges, $\mathrm{S}$ and $\mathrm{T}$, on the circumscribed circle pass through the fixed point $\mathrm{H}$ on the incident beam, so also on the ICS locus do the tangents that represent the reflected incident beam at the full optical scan angles $\pm A(\theta= \pm A / 2)$ (Figures 7.6, 7.7, and 7.8).

The ICS locus shown in Figure 7.8 displays the expected symmetry for an unlikely incident beam offset angle $2 \beta$ of zero degrees. The peak of the ICS cusp characteristic touches the inscribed circle of the polygonal scanner and the locus extends beyond the circumscribed circle.

Figure 7.9 shows the asymmetry of the ICS locus for a realistic incident beam offset angle $2 \beta$ of $70^{\circ}$. The peak of the ICS cusp characteristic lies within the inscribed circle of the polygonal scanner, while one extremity lies beyond the circumscribed circle and the other lies between the two circles. The tangent on the ICS locus at the data point $\theta=0^{\circ}$ corresponds to the scan-axis.

Figure 7.10 shows the asymmetry of the ICS locus for an incident beam offset angle $2 \beta$ of $100^{\circ}$. The peak of the ICS cusp characteristic lies within the inscribed circle of the polygonal scanner, as does one extremity $\theta=+30^{\circ}$, while the other extremity $\theta=-30^{\circ}$, lies between the inscribed and the circumscribed circles. The tangent on the ICS locus at the data point $\theta=0^{\circ}$ corresponds to the scan-axis.

Figure 7.11 shows a more extreme asymmetry of the ICS locus for an incident beam offset angle $2 \beta$ of $140^{\circ}$ for a 12 -facet polygonal scanner, $N=12$. The peak of the ICS cusp 
has disappeared because, in part, the range of the full mechanical scan angle $\theta$ has been reduced from $\pm 30^{\circ}$ to $\pm 15^{\circ}$ by virtue of the increased number of facets from six to twelve. The ICS locus extremities range from $\theta=+15^{\circ}$ within the inscribed circle of the polygonal scanner to $\theta=-15^{\circ}$ between the inscribed and the circumscribed circles. The tangent on the ICS locus at the data point $\theta=0^{\circ}$ corresponds to the scan-axis.

\subsubsection{Locus of $P$}

Axiomatically the locus of the point of incidence P lies along the incident beam line segment HP. Point $\mathrm{P}$ runs back and forth from the fixed point $\mathrm{H}$ on the incident beam, at which the circumference of the circumscribed circle of the polygonal scanner intersects. As the reflected incident beam scans through the full angular range $\pm A$ the locus of $\mathrm{P}$ overlaps itself.

The locus of $\mathrm{P}$ is inherently a straight line along the incident beam but doubles back on itself from and to the fixed point $\mathrm{H}$. To provide visibility of this locus the data ordinate $y / r$ values of Figure 7.10 have been mathematically and linearly stretched in Figure 7.12. For clarity the scale of the $x / r$ axis is significantly magnified about tenfold. The markedly different spacing between data plots at two-degree intervals is indicative of a rapid acceleration and slow deceleration as the point of incidence $\mathrm{P}$ traverses the facet.

\subsubsection{Offset Angle Limits}

The incident beam is not likely to lie within the scan angle when the plane of incidence is normal to the axis of rotation; therefore, the smallest offset angle $[2 \beta]_{\min }$ for a beam with an infinitesimal diameter will always be equal to, or greater than, the semi full optical scan angle $+A$.

The maximum offset angle $[2 \beta]_{\max }$ of the incident beam with an infinitesimal diameter occurs when the incident beam is at grazing incidence for the semi full optical scan angle $-A$. Therefore, the upper limit to the offset angle will always be equal to, or less than $\left(180^{\circ}-A\right)$.

Thus $2 \beta$ lies in the range

$$
\frac{360^{\circ}}{N} \leq 2 b \leq 180^{\circ}\left(1-\frac{2}{N}\right), \quad N \geq 4
$$

For real incident beams with a finite width (diameter) the minimum limit $360 \% \mathrm{~N}$ will increase and the maximum limit $180^{\circ}(1-2 / N)$ will decrease (see scan duty cycle $\eta$ in Sections 7.2.6 and 7.4.8).

The expressions for the limits of the offset angles provide a useful guideline in the design of a scanning system. Although one will endeavor to design the incident beam to have an offset angle $2 \beta$ to lie close to the semi full scan angle $+A$ of the reflected incident beam, there are occasions, for reasons of packaging, where this is impossible.

\subsubsection{Finite Beam Width $D$}

For simplicity the width of the beam has been assumed to be infinitesimal (Section 7.3.4) such that the objective lens optical axis is directed through the fixed point $\mathrm{H}$ on the incident beam where it is intersected by the circumscribed circle of the polygonal scanner in the midposition. If the incident beam has a finite width $D$, the radius $r$ in Equation 7.45 would be replaced by $r(1-g / r)$ because the incident beam shifts to the left to pass through 
the point G. The dimensional symbol " $g$ " is that shown in Figure 7.2 (Section 7.2). The $r$ in Equation 7.46 remains unchanged.

For a finite beam width $D$ the basic cusp-shape characteristic of the ICS loci that is shown in Figures 7.8 to 7.11 remains the same, but, with the exception of Figure 7.8 because of symmetry, it will be slightly displaced in an upward direction parallel to the facet plane by an amount $(g \tan \beta$ ) (Figure 7.2). The scan-axis is raised and the objective lens axis is lowered, each by an amount $(g \sin \beta)$ with respect to the coordinate axes $x / r$ and $y / r$. These displacement amounts are derived from Figure 7.2, Equations 7.21 and 7.15 in Sections 7.2.9 and 7.2 .8 , respectively.

\subsubsection{Commentary}

The ICS curves with offset angles greater than zero display interesting asymmetrical cusp-shape characteristics that offer potential insights into pupil movement that give rise to asymmetric aberrations in the image plane of pre-objective scanning systems.

\subsubsection{Conclusion}

An analysis of real beams of finite width (diameter) is fully expected to produce the same basic ICS characteristics as shown in the above documentation. The bottom line is that the ICS locus is of interest because it can give insight to the asymmetric wandering of the entrance pupil for the optical system lens designer who optimizes a design by minimizing the aberrations in the image plane regardless of the ICS locus.

\subsection{STATIONARY GHOST IMAGES OUTSIDE THE IMAGE FORMAT}

Ghost images are caused by both specular and scattered reflected rays from optical surfaces and are always unwanted, especially within the image format of the scanned field image plane. Various design innovations have been invented to minimize the effects or the presence of ghost images in the image format. Notable are those given in References 7 and 8 in which a limited angular range for the incident beam offset angle $(2 \beta)$ from the scan-axis is given so that stationary ghost images are formed outside the image format of the scanned field image plane of regular prismatic polygonal scanning systems. ${ }^{6}$

\subsubsection{Objective}

This section explores and illustrates the formation of stationary ghost images that are produced only by the scattered light rays from the scanned field image plane itself. The goal is to determine the angular ranges and limits of the incident beam offset angles $2 \beta$ (beyond that given in References 7 and 8 mentioned above), with visual insights that ensure that the stationary ghost images lie outside the image format.

\subsubsection{Stationary Ghost Images}

Ghost images in the image plane from nonmoving optical components may be expected, but, at first thought, not from a rotating optical component such as a polygonal scanner, and if so, certainly not stationary. However, the rotating polygonal scanner itself synchronously derotates (descans) these unwanted diffusely reflected rays from the image 
plane itself, and they are then specularly rereflected at the mirror facets. If these secondary specularly reflected rays are transmitted through the optics of the pre-objective optical scanning system, stationary ghost images will be formed in the image plane.

\subsubsection{Facet Angle $A$}

The facet angle $A$ is the angle that the facet subtends at the rotation axis $\mathrm{O}$ :

$$
A=\frac{360}{N}
$$

where $N$ represents the number of facets. For this section let $N=10$. Then,

$$
A=36^{\circ}, \text { and } 2 A=72^{\circ}
$$

\subsubsection{Facet-to-Facet Tangential Angle}

The mirror facet-to-facet tangential angle is the angle between successive facet normals in a plane perpendicular to the rotation axis. This angle is also denoted by the symbol $A$, because for a regular prismatic polygonal scanner the facet angle and the facet-to-facet tangential angle are geometrically identical.

\subsubsection{Scan-Axis}

The scan-axis is the axis about which the beam angularly scans symmetrically, $\pm A$ (see Sections 7.2.2 and 7.3.3).

\subsubsection{Offset Angle $2 \beta$}

The incident beam offset angle $2 \beta$ is the angle that the incident beam makes with the scanaxis.

\subsubsection{Midposition}

The midposition of a scanner is that orientation of the polygonal scanner for which a facet reflects the incident beam collinearly with the scan-axis, which is parallel to the objective lens optical axis (see Sections 7.2.2 and 7.3.3, and Figure 7.1).

\subsubsection{Scan Duty Cycle (Scan Efficiency) $\eta$}

The maximum potential scan duty cycle $\eta$ of a polygonal scanner is the ratio of the useful scan angle, during which the beam width $D$ is effectively unvignetted by the edges of the facets, to the full scan angle $\pm A$ of a beam with an infinitesimal width $(D=0)$. We shall assume that the footprint of the beam's tangential width is less than the facet's tangential width in the midposition of a polygonal scanner.

$$
h=1-\frac{\arcsin [D /(2 r \cos b)]}{180 / N}
$$


in which $r$ represents the radius of the circumscribed circle of the polygonal scanner (see Section 7.2.6). It can be seen from Equation 7.56 that for a given beam of finite width $D$ the scan duty cycle $\eta$ decreases with an increase in the offset angle $2 \beta$, or with an increase in the number of facets $N$.

\subsubsection{Rotation Axis Offset Distance}

The rotation axis offset distance is that distance $Y_{P}$ of the rotation axis from the scan-axis when the polygonal scanner is set in its midposition (Figures 7.2 and 7.3).

The rotation axis offset distance $Y_{P}$ depends on the number of facets $N$, the incident beam offset angle $2 \beta$, and beam width $D$. Replicating Equation 7.25 with a negative sign from Section 7.2 gives

$$
Y_{P}=-r \sin b\left[2 \cos \left(\frac{180}{N}\right)\right]-\sqrt{\left[1-\left(\frac{D}{2 r \cos b}\right)^{2}\right]}
$$

in which $r$ again represents the radius of the circumscribed circle of the polygonal scanner.

Alternatively, replicating Equation 7.27 embodying in the scan duty cycle $\eta$ from Section 7.2 leads to

$$
Y_{P}=-r \sin b\left[2 \cos \left(\frac{180}{N}\right)\right]-\sqrt{\left(1-\left\{\sin \left[\left(\frac{180}{N}\right)(1-h)\right]\right\}^{2}\right)}
$$

For an infinitesimal beam width $(D=0)$ or a $100 \%$ scan duty cycle $(\eta=1)$, Equations 7.57 and 7.58 both reduce to

$$
Y_{P}=-r \sin b\left[2 \cos \left(\frac{180}{N}\right)-1\right], \quad N \geq 3
$$

It can be seen from Equation 7.59, Table 7.3, and supported by Figures 7.13 to 7.16, that as the incident beam offset angle $2 \beta$ increases, and/or the number of facets $N$ increases, so also does the rotation axis offset distance increase $Y_{P}$.

When $(N=3)$, Equation 7.59 leads to $Y_{P}=0$, which means the rotation axis lies on the scan-axis (Figure 7.4 and Section 7.2.13).

TABLE 7.3

Incident Beam Offset Angle $2 \beta$ versus Maximum Potential Scan Duty Cycle $\eta$

\begin{tabular}{lccr}
\hline $\begin{array}{l}\text { Incident Beam } \\
\text { Offset Angle, } \boldsymbol{\beta} \boldsymbol{\beta}\end{array}$ & $\begin{array}{c}\text { Maximum Potential } \\
\text { Scan Duty Cycle, } \boldsymbol{\eta}\end{array}$ & $\begin{array}{c}\text { Rotation Axis Offset Distance, } \boldsymbol{Y}_{\boldsymbol{P}} \\
\text { Distance from the Scan Axis }\end{array}$ & Figure \\
\hline $27^{\circ}$ & $93.5 \%$ & $-0.211 r$ & 7.13 \\
$52^{\circ}$ & $92.9 \%$ & $-0.395 r$ & 7.14 \\
$92^{\circ}$ & $90.8 \%$ & $-0.649 r$ & 7.15 \\
$124^{\circ}$ & $86.4 \%$ & $-0.797 r$ & 7.16 \\
$164^{\circ}$ & $54.1 \%$ & $-0.904 r$ & - \\
\hline
\end{tabular}




\subsubsection{Choosing an Incident Beam Offset Angle 2 $\beta$}

Ideally, for symmetry of design, the incident beam should be directed along the scan-axis, but this would obstruct the reflected scanning beam. Therefore, if the image format field angle is $2 \omega$, then the incident beam offset angle $2 \beta$ must at least be slightly greater than the semi-image format field angle $\omega$ to avoid this physical interference (Figure 7.13). That is,

$$
2 b>w
$$

Using Equations 7.56 and 7.57 with $N=10, r=25 \mathrm{~mm}$, and $D=1 \mathrm{~mm}$, leads to Table 7.3.

\subsubsection{Ghost Beams gh and Images GH}

Pencils of scattered light rays from the incident scanning spot on the scanned surface plane are returned through the objective lens. The objective lens recollimates them as they proceed back to the polygonal scanner's facets, at which they are specularly reflected to produce what are known as ghost beams. In Figures 7.13 through 7.16 these ghost beams are symbolized by the letters gh, with a subscript that identifies the facet whence they came.

Only if the ghost beams gh are reflected from a facet of the polygonal scanner at angles numerically much less than $90^{\circ}$, that is, toward the objective lens, is there a chance that they can traverse back through the objective lens, which will focus them onto the image plane to form stationary point ghost images GH. A subscript to GH refers to the respective facet whence the ghost beam gh came.

If these ghost beams are reflected from a facet of the polygonal scanner at angles numerically greater than $90^{\circ}$, that is, away from the objective lens, there is no chance of them traversing back through the objective lens to produce stationary ghost images GH.

\subsubsection{Ghost Beam Field Angles $\phi$}

The field angle $\varphi$ of all ghost beams gh, whether they produce stationary ghost images in the image format plane or not, is always at an angle that is a multiple of $2 A$ away from, and on either side of, the incident beam offset angle $2 \beta$. This is because the mirror facet-to-facet tangential angle of a regular prismatic polygonal scanner is $A$. Thus

$$
f=2 b \pm n(2 A), \quad|2 b \pm n(2 A)|<90^{\circ}
$$

in which $n$ is an integer.

In Section 7.4.3, $N=10$, therefore $24=72^{\circ}$; hence all ghost beams in Figures 7.13 through 7.16 occur at intervals of $72^{\circ}$ from the incident beam offset angle $2 \beta$.

When $n=0$, Equation 7.61 represents the retroreflective ghost beam field angle $\phi_{1}$ that is collinear with the incident beam. Ghost beam $\mathrm{gh}_{1}$ is not relevant in this discussion and, to avoid confusion, it is not depicted in the figures.

If one increases the incident beam offset angle $2 \beta$ by say $25^{\circ}$ counterclockwise, and repositions the polygonal scanner to a midposition, all the field angles $\phi$ of the ghost beams will have also rotated by $25^{\circ}$ counterclockwise, while the polygonal scanner will have only rotated $12.5^{\circ}$ counterclockwise; and vice versa, if clockwise.

\subsubsection{Incident Beam Location}

Figures 7.13 through 7.16, which shall be discussed in turn, depict the first significant four incidence beam offset angular positions given in Table 7.3 , namely, $27^{\circ}, 52^{\circ}, 92^{\circ}$, and $124^{\circ}$. 
Figures 7.13 through 7.16 depict the respective orientations of the polygonal scanner in the midposition and the rotation axis offset distances $Y_{P}$, such that the reflected incident beam is collinear with the scan-axis to focus it to the central point $C$ in the image field format. Pencils of light rays, $\mathrm{gh}_{2}, \mathrm{gh}_{3}, \mathrm{gh}_{4}$, and $\mathrm{gh}_{10}$, scattered from point $\mathrm{C}$, are shown passing back through the objective lens to meet the facets of the polygonal scanner, whence they are again reflected. The subscripts correspond to the facet number.

In Figure 7.13 one such pencil, $\mathrm{gh}_{2}$, passes back through the objective lens to produce the point image $\mathrm{GH}_{2}$ below the image field format. There is one pencil $\mathrm{gh}_{1}$, which is reflected from facet $S_{1}$ that is not displayed so as not to overcrowd the diagram. Pencil $\mathrm{gh}_{1}$ is the retroreflective pencil that returns collinearly with the path of the incident beam.

As predicted by Equation 7.61 the angle between successive pencils of rays of ghost beams gh reflected from the five facets $S_{10}, S_{1}, S_{2}, S_{3}$, and $S_{4}$ is $2 A$ (Figures 7.13 to 7.16$)$.

One should notice that in Figures 7.13 to 7.16 the vertices of the fan depicting the full scan angle $\pm A$ and the image field format scan angle $\pm \omega$ do not coincide, nor do they touch the surface of the facet. They lie at two distinct locations. The first lies on the incident beam axis at its intersection with the circumscribed circle of the polygonal scanner; the second lies below, within the circumscribed circle and above the scan-axis. The difference is best observed in Figure 7.16 (see also Section 7.3).

\subsubsection{Image Format Scan Duty Cycle $\eta_{\omega}$}

The image format scan duty cycle $\eta_{\omega}$, is the ratio of the image field format angle $2 \omega$ to the full scan angle $2 A$ of the polygonal scanner. It must not be confused with the maximum potential scan duty cycle (scan efficiency) $\eta$. The image format scan duty cycle $\eta_{\omega}$ depends directly on the image format angle $2 \omega$.

$$
h_{w}=\frac{2 w}{2 A}=\frac{w}{A}
$$

In Figures 7.13 to $7.16,2 \omega= \pm 20^{\circ}\left(40^{\circ}\right)$. Substituting for $\omega$ and $A$ into Equation 7.62 leads to

$$
h_{W}=\frac{20^{\circ}}{36^{\circ}}=55.6 \%
$$

Since the above image field format scan duty cycle $\eta_{\omega}$ of $55.6 \%$ is greater than the maximum potential scan duty cycle $\eta$ of $54.1 \%$ presented in Table 7.3 for an incident beam offset angle $2 \beta$ of $164^{\circ}$, this offset angle is not relevant and no figure is provided. The image field format scan duty cycle $\eta_{\omega}$ must be less than the scan duty cycle $\eta$.

\subsubsection{Incident Beam Offset Angle 27}

The incident beam offset angle of $27^{\circ}$ in Figure 7.13 is comfortably outside the semi-image format angle $\omega$ of $+20^{\circ}$ to avoid physical obstruction of the scanning beam, but at an angle less than the half scan angle $A=+36^{\circ}$, of the ten-facet polygonal scanner. 


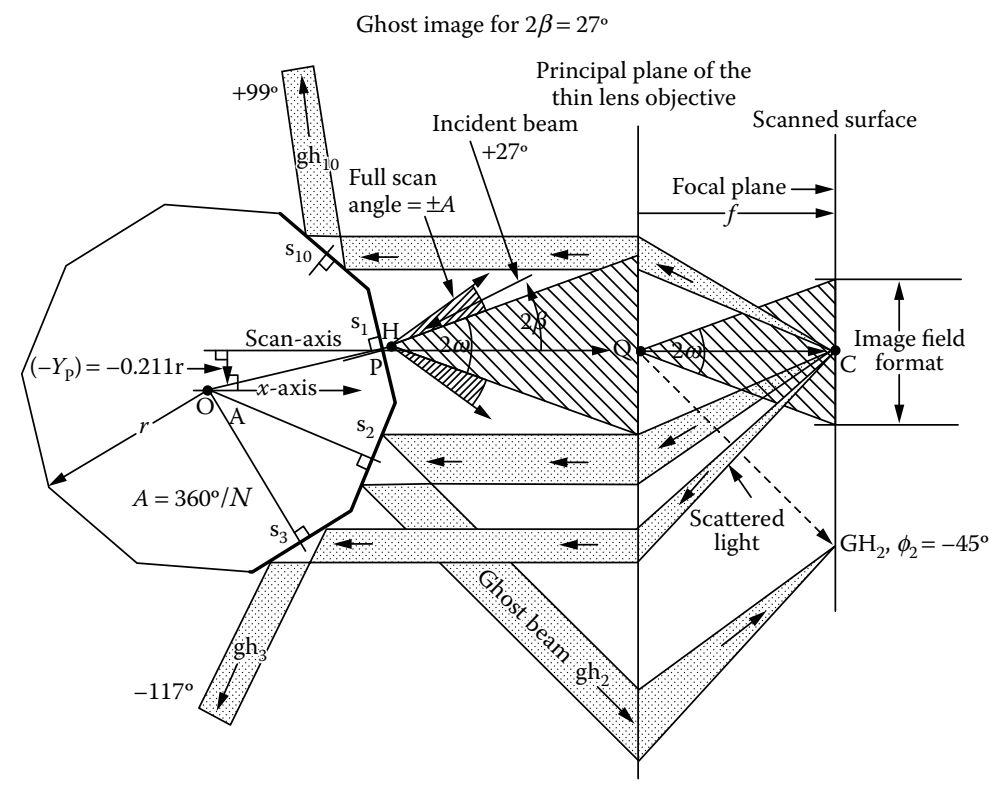

FIGURE 7.13

Formation of stationary ghost image $\mathrm{GH}_{2}$ is produced by a pencil of scattered rays originating at $\mathrm{C}$ and rereflected from facet $S_{2}$ at a field angle $\phi_{2}=-45^{\circ}$.

If the ghost beam $\mathrm{gh}_{2}$ traverses the objective lens, there is only one stationary ghost image, $\mathrm{GH}_{2}$, at a field angle of $\phi_{2}=-45^{\circ}$; and it lies outside and $25^{\circ}$ below the image field format.

From Equation 7.61 the field angles of ghost beams $\mathrm{gh}_{10}$ and $\mathrm{gh}_{3}$ from facet $\mathrm{S}_{10}$ and $\mathrm{S}_{3}$, are $\phi_{10}=+99^{\circ}$ and $\phi_{3}=-117^{\circ}$, respectively. These ghost beams are harmless $\left(|f|>90^{\circ}\right)$.

\subsubsection{Incident Beam Offset Angle 52 ${ }^{\circ}$}

In Figure 7.13 the incident beam offset angle is $27^{\circ}$. Let the incident beam offset angle $2 \beta$ with its accompanying ghost beams gh and ghost images $\mathrm{GH}$ be rotated counterclockwise through a positive angle of $+25^{\circ}$. If the ghost beam $\mathrm{gh}_{2}$ traverses the objective lens, the ghost image $\mathrm{GH}_{2}, \phi_{2}=-45^{\circ}$, of Figure 7.13 will move up to lie on the lower edge $\left(\nabla \omega=\nabla 20^{\circ}\right)$ of the image format field, $\phi_{2}=\left(-45^{\circ}+25^{\circ}\right)=\bigotimes 20^{\circ}$. The incident beam offset angle increases to $2 b=\left(+27^{\circ}+25^{\circ}\right)=+52^{\circ}$ (Figure 7.14).

From Equation 7.61 the field angles of ghost beams $\mathrm{gh}_{10}$ and $\mathrm{gh}_{3}$ from facets $\mathrm{s}_{10}$ and $\mathrm{s}_{3}$ become $\phi_{10}=+124^{\circ}$ and $\phi_{3}=\nabla 92^{\circ}$, respectively. These ghost beams are harmless $\left(|f|>90^{\circ}\right)$.

\subsubsection{Incident Beam Offset Angle 92 ${ }^{\circ}$}

In Figure 7.14 the incident beam offset angle is $52^{\circ}$. Let the incident beam offset angle $2 \beta$ with its accompanying ghost beams gh and ghost images $\mathrm{GH}$ be rotated counterclockwise through a positive angle of $+40^{\circ}$. If the ghost beam $\mathrm{gh}_{2}$ traverses the objective lens, the ghost image $\mathrm{GH}_{2},\left(\phi_{2}=\nabla \omega=\nabla 20^{\circ}\right)$, of Figure 7.13 will move up to lie on the upper edge 


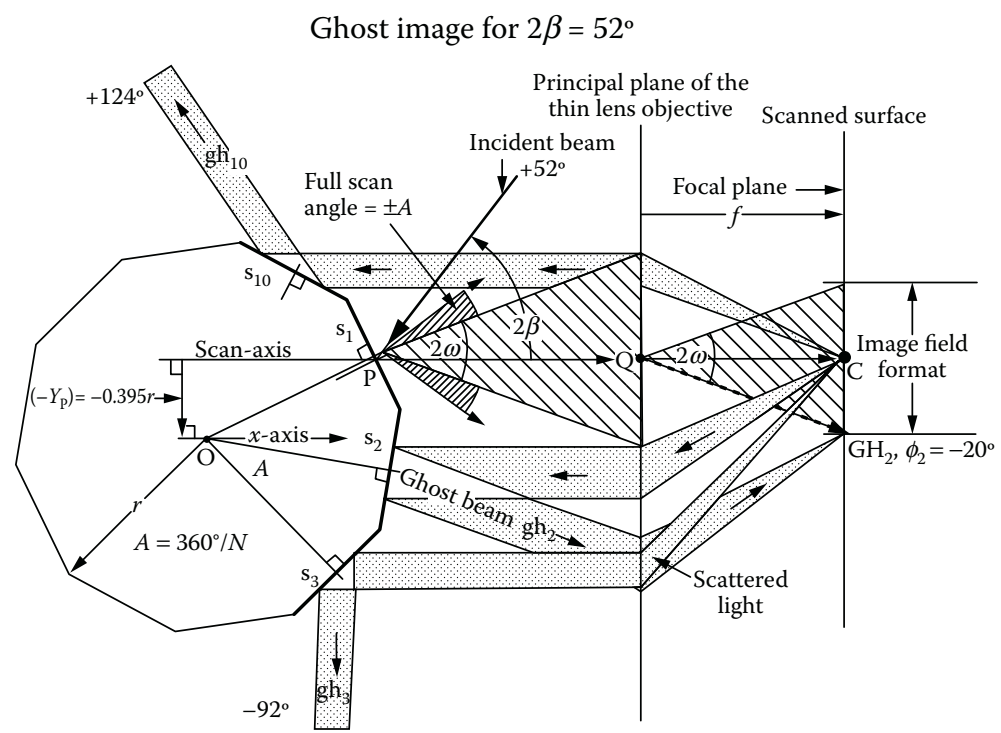

\section{FIGURE 7.14}

The stationary ghost image $\mathrm{GH}_{2}$ at the lower edge of the image field format at a field angle $\phi_{2}=\bigotimes 20^{\circ}$ is produced by a pencil of scattered rays originating at $C$ and rereflected from facet $S_{2}$.

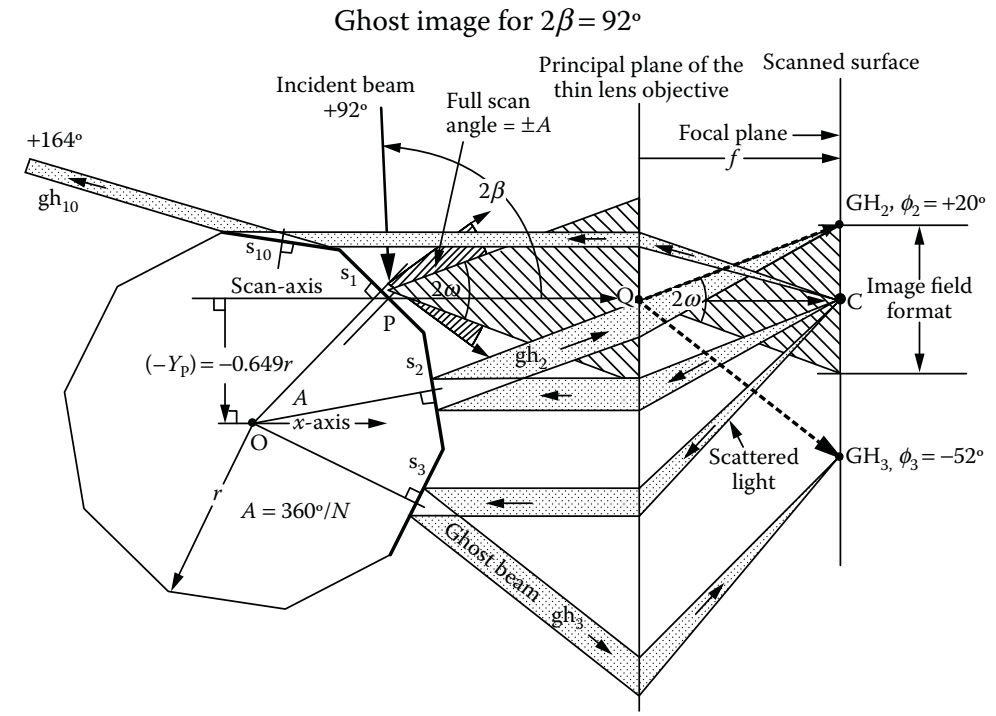

\section{FIGURE 7.15}

If the ghost beams $\mathrm{gh}_{2}$ and $\mathrm{gh}_{3}$ traverse the objective lens, there is a stationary ghost image $\mathrm{GH}_{2}$ at the upper edge of the image field format $\phi_{2}=+20^{\circ}$ and a stationary ghost image $\mathrm{GH}_{3}$ below it, $\phi_{3}=\varangle 52^{\circ}$.

$\left(+\omega=+20^{\circ}\right)$ of the image format field, $\phi_{2}=\left(\nabla \omega+40^{\circ}\right)=\left(\nabla 20^{\circ}+40^{\circ}\right)=+20^{\circ}$. The incident beam offset angle increases to $2 b=\left(+52^{\circ}+40^{\circ}\right)=92^{\circ}$ (Figure 7.15).

For incident beam offset angles $27^{\circ}$ and $52^{\circ}$ there is only one stationary ghost image in the image format, namely $\mathrm{GH}_{2}$. As ghost image $\mathrm{GH}_{2}$ moves to the upper edge of the image format, a second ghost image $\mathrm{GH}_{3}$ appears in the image format well below at a field angle, $\phi_{3}=\bigotimes 52^{\circ}$. Note that $\left(\phi_{2} \otimes \phi_{3}\right)=2 A=72^{\circ}$, as expected. 
From Equation 7.61 the field angle of the remaining ghost beam $\mathrm{gh}_{10}$ from facet $\mathrm{s}_{10}$ is $\phi_{10}=+164^{\circ}$, and is harmless $\left(|f|>90^{\circ}\right)$.

\subsubsection{Incident Beam Offset Angle $124^{\circ}$}

In Figure 7.15 the incident beam offset angle is $92^{\circ}$. Let the incident beam offset angle $2 \beta$ with its accompanying ghost beams gh and ghost images $\mathrm{GH}$ be rotated counterclockwise through a positive angle of $+32^{\circ}$. If the ghost beam $\mathrm{gh}_{3}$ traverses the objective lens, the ghost image $\mathrm{GH}_{3}, \phi_{3}=\nabla 52^{\circ}$, of Figure 7.15 will move up to lie on the lower edge $\left(\nabla \omega=\nabla 20^{\circ}\right)$ of the image format field, $\phi_{3}=\left(\bigotimes 52^{\circ}+32^{\circ}\right)=\bigotimes 20^{\circ}$. The incident beam offset angle increases to $2 b=\left(+92^{\circ}+32^{\circ}\right)=+124^{\circ}$ (Figure 7.16).

For incident beam offset angles $52^{\circ}$ and $92^{\circ}$ there are two stationary ghost images, namely $\mathrm{GH}_{2}$ and $\mathrm{GH}_{3}$. As ghost image $\mathrm{GH}_{3}$ moves to the lower edge of the image format, ghost image $\mathrm{GH}_{2}$ moves up well above the image format at a field angle, $\phi_{2}=$ $+52^{\circ}$. Again note that $\left(f_{2}-f_{3}\right)=2 A=72^{\circ}$, as should be expected. From Equation 7.61 the field angle of the remaining ghost beam $\mathrm{gh}_{4}$ from facet $\mathrm{s}_{4}$ is $\phi_{4}=\nabla 92^{\circ}$, and is harmless $\left(|f|>90^{\circ}\right)$.

A simple calculation of adding $72^{\circ}$ to the field angle $\phi_{10}=164^{\circ}$ of ghost beam $\mathrm{gh}_{10}$ in Figure 7.15 produces a reflex angle of $236^{\circ}$, thus predicting that the ghost beam $\mathrm{gh}_{10}$ can no longer exist.

A close inspection of Figures 7.13 to 7.16 shows the center-of-scan of the total angular scan $2 A$ of the scanner progressively becomes displaced from the center-of-scan of the image format scan angle $2 \omega$ with an increase in the incident beam offset angle $\beta$. This is valid because the ICS is a locus (see Section 7.3 and Reference 5).

Ghost image for $2 \beta=124^{\circ}$

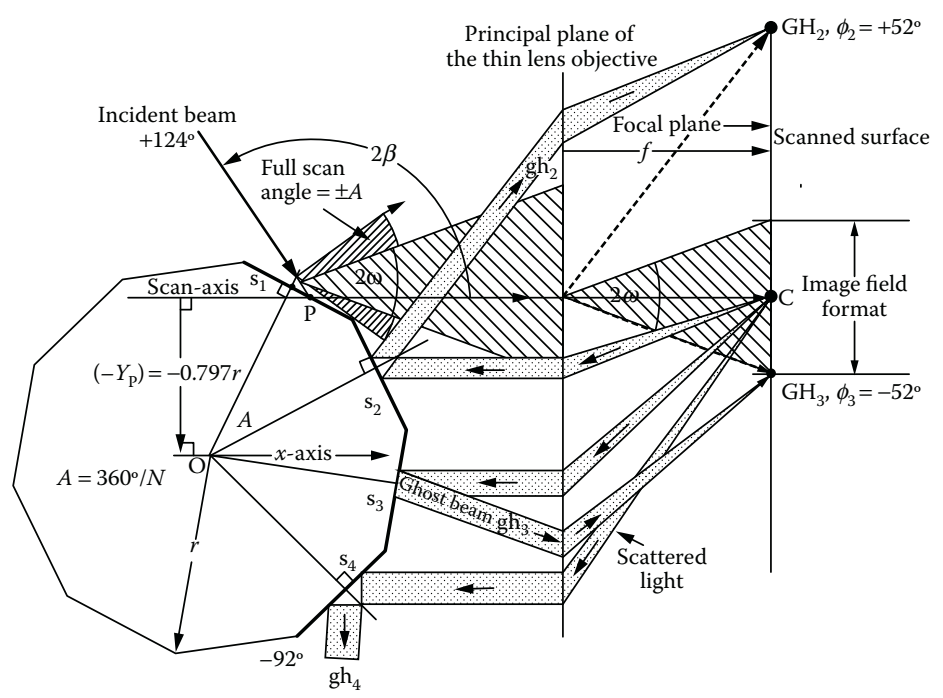

FIGURE 7.16

If the ghost beams $\mathrm{gh}_{2}$ and $\mathrm{gh}_{3}$ traverse the objective lens, there is a stationary ghost image $\mathrm{GH}_{3}$ at the lower edge of the image field format $\phi_{3}=\nabla \omega=\nabla 20^{\circ}$ and a stationary ghost image $\mathrm{GH}_{2}$ above, $\phi_{2}=+52^{\circ}$. 


\subsubsection{Ghost Images Inside the Image Format}

A study of Figures 7.14 and 7.15 shows that, if ghost image $\mathrm{GH}_{2}$ is set on the lower and upper edges of the image format, the required incident beam offset angles are given by

$$
2 b=2 A+w
$$

Thus, from Equation 7.61 the range of $2 \beta$ for ghost images to exist inside the image format is expressed by

$$
n(2 A)-w<2 b<n(2 A)+w
$$

in which $n$ is zero or a positive integer, $A \geq \omega$, and $2 \beta<180^{\circ}$.

Substituting $\omega=20^{\circ}$ and $2 A=72^{\circ}$ leads to

$$
\begin{array}{ll}
\text { when } n=0 & -20^{\circ}<2 b<+20^{\circ} \\
n=1 & +52^{\circ}<2 b<+92^{\circ} \\
n & =2+124^{\circ}<2 b<+164^{\circ}
\end{array}
$$

Each has a range of $40^{\circ}$, which, not surprisingly, equates to $2 \omega$.

A figure showing $2 \beta=164^{\circ}$ is not relevant or depicted, because it has a scan duty cycle $\eta$ less than the required image format duty cycle $\eta_{\omega}$ (Table 7.3).

\subsubsection{Ghost Images Outside the Image Format}

A study of expressions (7.66), (7.67), and (7.68) shows that when the incident beam offset angle lies between $+20^{\circ}$ and $+52^{\circ}$ no ghost image will appear in the image format. Likewise, when the incident beam offset angle lies between $+92^{\circ}$ and $+124^{\circ}$, each has a range of $32^{\circ} .^{2}$

Thus, to ensure ghost images lie outside the image format the condition is as follows:

$$
n(2 A)+w<2 b<(n+1)(2 A)-w
$$

in which $n$ is zero or a positive integer, $\mathrm{A} \geq \omega$, and $2 \beta<180^{\circ}$.

Let $\rho$ represent the angular range of $2 \beta$ for ghost images outside the image format, then

$$
r=2 A-2 w=2(A-w)=2(180 / N-w)
$$

and is independent of $n$.

\subsubsection{Number of Facets}

Subject toA $\geq \omega$, as the number of facets $N$ increases, so also does the number of ghost beams gh and, therefore, there is a greater possibility of multiple ghost images $\mathrm{GH}$ in the scanned image plane. A critical case, in this example, occurs when $N=18$. Then $A=+\omega=+20^{\circ}$. 
Substituting these values for $A$ and $\omega$ into the inequalities (69) and (70) leads to Figure 7.17.

$$
\text { For } \begin{aligned}
n=0, & 20^{\circ}<2 b<20^{\circ} \\
n & =1, \quad 60^{\circ}<2 b<60^{\circ} \\
n & =2, \quad 100^{\circ}<2 b<100^{\circ}
\end{aligned}
$$

and the range

$$
r=0^{\circ}
$$

Hence, the positioning tolerance for the incident beam offset angle $2 \beta$ is zero. For an adequate positioning tolerance for the incident beam

$$
A>W
$$

Substituting $A=360 \% \mathrm{~N}$ leads to the general condition

$$
\frac{360}{N}>w
$$
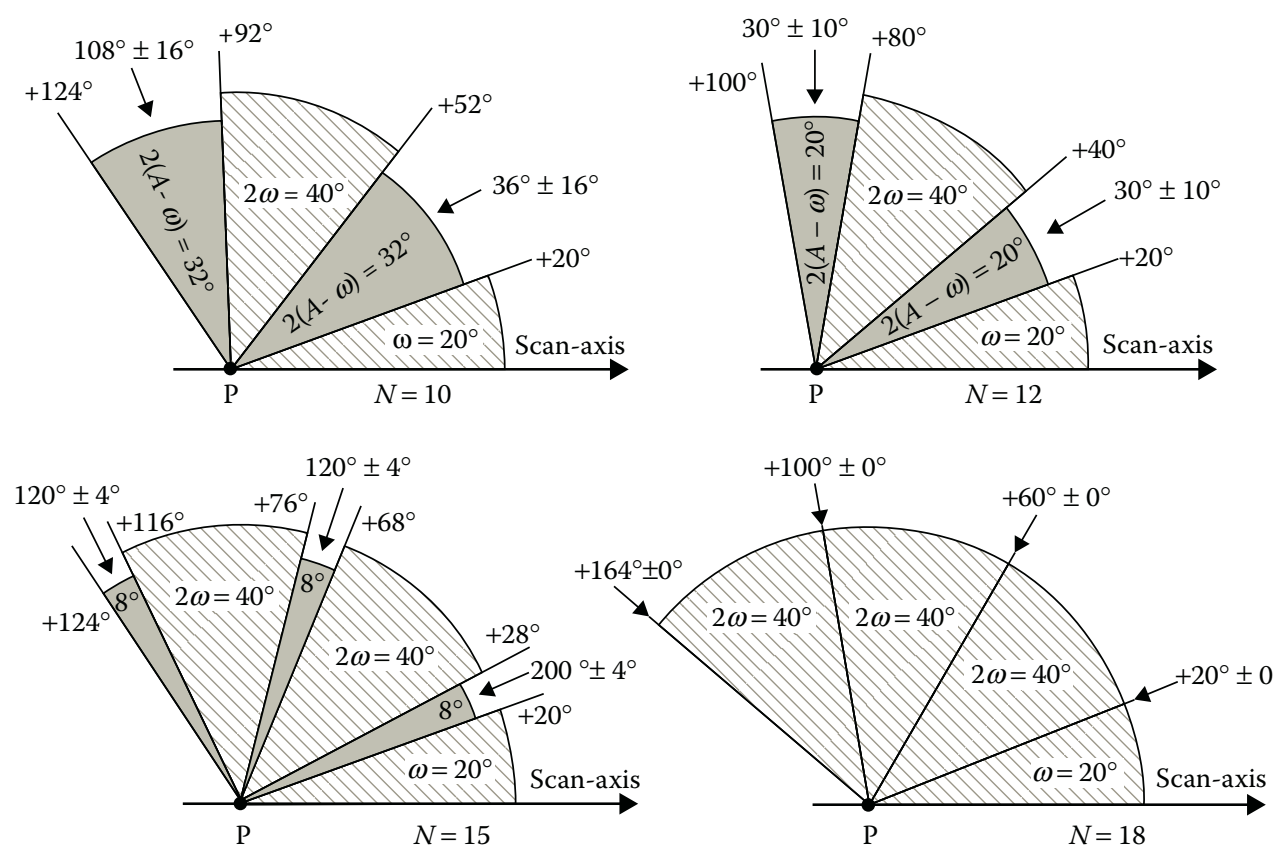

\section{FIGURE 7.17}

To ensure that stationary ghost images GH are outside the image format, the angular ranges $\rho$ of the incident beam offset angle $2 \beta$ are shown at $32^{\circ}, 20^{\circ}, 8^{\circ}$, and $0^{\circ}$. The illustration also shows the angular ranges $\rho$ for 10 -facet, 12 -facet, 15 -facet, and 18-facet polygonal scanners. 
In Figure 7.17, P represents the point of incidence on the scanner facet. The image format field angle shown is $2 \omega=40^{\circ}$. For the 18 -facet polygon the angular range is zero, but theoretically available, and would simultaneously produce ghost images $\mathrm{GH}_{2}$ and $\mathrm{GH}_{18}=$ at the upper, $\phi_{2}=+\omega$, and lower, $\phi_{18}=\nabla \omega$, edges of the image format, respectively, when the incident beam offset angle is $2 b=+w,+3 w,+5 w,+7 w$, and so on, subject to $2 \beta<180^{\circ}$.

\subsubsection{Diameters of Scanner and Objective Lens}

No mention has been made with respect to the diameters of the objective lens, the scanner, nor the apertures near the scanner, or performance. These topics are out of the scope of this section, but all are important issues. ${ }^{2}$

However, the smaller the diameter of the scanner relative to the objective lens diameter, the greater the chance of a ghost beam returning to produce a ghost image in the scanned image plane. Likewise, the closer the scanner is to the objective lens, the greater the chance of a ghost beam returning to produce a ghost image in the scanned image plane.

\subsubsection{Commentary}

There is more than one angular zone for the incident beam offset angle to avoid ghost images appearing within the image format. These zones have acceptable scan duty cycles $\eta$, depending on beam width $D$, the diameter $2 r$ of the polygonal scanner and the number of facets $N$ (Figure 7.17, Table 7.3). ${ }^{2}$

\subsubsection{Conclusion}

It behooves one to consider the possibility and the whereabouts of stationary ghost images in the image format plane during the initial optical system design stage.

\section{ACKNOWLEDGMENTS}

The author appreciates the time and expertise that Leo Beiser and Stephen Sagan have given in reviewing this chapter and providing many helpful suggestions. I thank the optomechanical design engineers of CSIRO, Australia, who encouraged me to solve and provide the explicit "Coordinates and Equations of a Polygonal Scanning System" for a beam with a finite width as presented in Section 7.2.

\section{REFERENCES}

1. Kessler, D.; DeJaeger, D.; Noethen, M. High resolution laser writer. Proc. SPIE, 1989, 1079, 27-35.

2. Beiser, L. Unified Optical Scanning Technology; IEEE Press, Wiley-Interscience, John Wiley \& Sons: New York, 2003. 
3. Beiser, L. Design equations for a polygon laser scanner. In Beam Deflection and Scanning Technologies; Marshall, G.F.; Beiser, L., Eds.; Proc. SPIE 1991, 1454, 60-66.

4. Marshall, G.F. Geometrical determination of the positional relationship between the incident beam, the scan-axis, and the rotation axis of a prismatic polygonal scanner. In Optical Scanning 2002; Sagan, S.F.; Marshall, G.F.; Beiser, L., Eds.; Proc. SPIE 2002, 4773, 38-51.

5. Marshall, G.F. Center-of-scan locus of an oscillating or rotating mirror. In Recording Systems: High-Resolution Cameras and Recording Devices; Laser Scanning and Recording Systems; Beiser, L.; Lenz, R.K., Eds.; Proc. SPIE 1993, 1987, 221-232.

6. Marshall, G.F. Stationary ghost images outside the image format of the scanned image plane. In Optical Scanning 2002; Sagan, S.F.; Marshall, G.F.; Beiser, L., Eds.; Proc. SPIE 4773, 132-140.

7. U.S. patent no. 5,191,463, 1990. Scanning optical system, in which ghost image is eliminated.

8. U.S. patent no. 4,993,792, 1986. Scanning optical system, in which ghost image is eliminated. 